\title{
Perturbative method for generalized spectral decompositions.
}

\author{
Roberto Laura \\ Departamento de Física, F.C.E.I.A., Universidad Nacional de Rosario. \\ Instituto de Física Rosario, CONICET-UNR \\ Av. Pellegrini 250, 2000 Rosario, Argentina. \\ e-mail: laura@ifir.ifir.edu.ar \\ Mario Castagnino \\ Instituto de Astronomía y Física del Espacio, \\ Casilla de Correos 67, Sucursal 28, \\ 1428 Buenos Aires, Argentina. \\ e-mail: castagni@iafe.uba.ar \\ Rodolfo M. Id Betan \\ Departamento de Física, F.C.E.I.A., Universidad Nacional de Rosario. \\ Instituto de Física Rosario, CONICET-UNR \\ Av. Pellegrini 250, 2000 Rosario, Argentina. \\ e-mail: rodolfo@ifir.ifir.edu.ar
}

(November 1998)

\begin{abstract}
Imposing analytic properties to states and observables we construct a perturbative method to obtain a generalized biorthogonal system of eigenvalues and eigenvectors for quantum unstable systems. A decay process can be described using this generalized spectral decomposition, and the final generalized state is obtained.
\end{abstract}

\section{INTRODUCTION.}

Following previous ideas about the use of analytic continuation in the study of unstable quantum systems [1], [2], [3], [4], in papers [5], [6] and [7] we have developed our own version, obtaining new results. But, as there were not a perturbative method adapted to our formalism, we were forced to study only solvable simple models. Therefore, this paper is devoted to fill this gap introducing a perturbation method adapted to our formalism. Let us briefly review the problem and the history of the proposed solution.

The decay of unstable systems in quantum mechanics is usually described by a Hamiltonian of the form $H=$ $H_{\text {free }}+H_{\text {int }}$, where $H_{\text {free }}$ has a discrete spectrum imbedded in the continuous spectrum, and $H$ has a continuous spectrum. In non equilibrium quantum statistical mechanics, the generator of the time evolution is the Liouville-Von Newmann operator which in some cases can be written as $\mathbb{L}=\mathbb{L}_{\text {free }}+\mathbb{L}_{\text {int }}$. There is an overlapping between the continuous and the discrete part of the spectrum of $\mathbb{L}_{\text {free }}$, where in this case the discrete part of the spectrum contains the zero eigenvalue of the generator of the free evolution. It is expected that the infinite dimensional degeneration of the zero eigenvalue to be partially removed by the interaction, so that the zero eigenspace of $\mathbb{L}$ turns out to be the equilibrium subspace.

E.g., for the Friedrichs model, a prototype of a decay system in quantum mechanics, with Hamiltonian

$$
H=\Omega|1\rangle\left\langle 1\left|+\int_{0}^{\infty} d \omega \omega\right| \omega\right\rangle\langle\omega|+\int_{0}^{\infty} d \omega V_{\omega}\{|\omega\rangle\langle 1|+| 1\rangle\langle\omega|\}, \quad \Omega \in \mathbb{R}^{+}, V_{\omega}=\overline{V_{\omega}},
$$

it is possible to find conditions on the interaction $V_{\omega}$ for which the spectrum of $H$ is $\mathbb{R}^{+}$. The discrete part of the spectrum of $H_{0}=\Omega|1\rangle\left\langle 1\left|+\int_{0}^{\infty} d \omega \omega\right| \omega\right\rangle\langle\omega|$ is eliminated by the interaction. A complete set of generalized right (left) eigenvectors $\left|\omega^{+}\right\rangle\left(\left\langle\omega^{+}\right|\right)$of the Hamiltonian $H$ with eigenvalue $\omega \in \mathbb{R}^{+}$can be explicitly obtained, thus $H=\int_{0}^{\infty} d \omega \omega\left|\omega^{+}\right\rangle\left\langle\omega^{+}\right|$. When $V_{\omega} \rightarrow 0,\left|\omega^{+}\right\rangle$tends to $|\omega\rangle$ and therefore the discrete eigenvector $|1\rangle$ is not recovered.

E.C.G.Sudarshan et al [1] obtained a generalized spectral decomposition for the Friedrichs model by considering a Hamiltonian obtained from the previous expression by replacing the integrals over $\mathbb{R}^{+}$by integrals over a curve $\Gamma$ in the lower complex half plane. This extended Hamiltonian $H_{\Gamma}$ is meaningful as the generator of the time evolution for state vectors $\Phi$ for which $\langle\omega \mid \Phi\rangle$ has a well defined analytic extension to the lower complex half plane. The spectrum of $H_{\Gamma}$ is the curve $\Gamma$ and a point $z_{0}$ located in the lower half plane between the curve $\Gamma$ and the real axis. When $V_{\omega} \rightarrow 0, z_{0}$ tends to $\Omega$, and the discrete part of the spectrum of the "free" Hamiltonian is recovered (see also reference [5]). 
The same complex spectral decomposition for the Friedrichs model was also obtained by T.Petrosky, I.Prigogine and S.Tasaki [8], with a perturbative method using a "time ordering rule" by which an small imaginary part is included to avoid small denominators, with a different sign according to the type of transition involved.

Latter, I.Antoniou et.al. [9], developed a formalism to deal with singular observables and generalized states, which they applied in the framework of the subdynamics theory. We gave our version of this formalism in papers [6] and [8]. We also considered singular observables and generalized states to describe the time evolution of a quantum oscillator interacting with a field, describing the evolution of the system to equilibrium in the thermodynamic limit [7].

The analytic dilation method [10] [11] is also a well established procedure to determine the contributions of the singularities to the time evolution of unstable quantum systems.

In this paper we consider states and observables with suitable analytic properties (as in papers [5] and [6]), such that the integrals over the continuous part of the spectrum can be deformed into integrals over curves in the complex plane. The physical motivation of these analytic properties is explained in [0]. In this way we can avoid the ill defined terms involving the differences between discrete and continuous eigenvalues of the unperturbed generator of the time evolution, and implement a well defined algorithm to obtain a complete biorthogonal set of generalized eigenvectors in one to one correspondence with the basis of the unperturbed problem. This biorthogonal system can be ued to give a description of the decay process.

The paper is organized as follows:

In section II we present the perturbative algorithm for the case of pure states, compute the complete set of right and left generalized eigenvectors and discuss the roll of the complex eigenvalues in the approximated expressions for the decay process. The method is applied to study the tunneling through a barrier in a one dimensional problem and to the Friedrichs model, were the results are compared with the exact solutions of reference [1]. In section III the case of generalized states and singular observables for the Friedrichs model is studied with the perturbative method. In the conclusions of section IV we summarize the results and discuss the physical interpretation of the complex generalized spectral decomposition.

\section{PURE STATES.}

Let us consider a system where the free Hamiltonian $\stackrel{0}{H}$ has a continuous spectrum plus a single discrete eigenvalue, and there is a general interaction $\stackrel{1}{H}$ :

$$
\begin{aligned}
& H=\stackrel{0}{H}+\stackrel{1}{H} \\
& \stackrel{0}{H} \doteq \Omega|1\rangle\left\langle 1\left|+\int_{0}^{\infty} d \omega \omega\right| \omega\right\rangle\langle\omega| \quad \Omega \in \mathbb{R}^{+}, \\
& \stackrel{1}{H} \doteq \int_{0}^{\infty} d \omega V_{\omega}|\omega\rangle\left\langle 1\left|+\int_{0}^{\infty} d \omega \overline{V_{\omega}}\right| 1\right\rangle\left\langle\omega\left|+\int_{0}^{\infty} d \omega \int_{0}^{\infty} d \omega^{\prime} V_{\omega \omega^{\prime}}\right| \omega\right\rangle\left\langle\omega^{\prime}\right|, \quad V_{\omega \omega^{\prime}}=\overline{V_{\omega^{\prime} \omega}}, \quad \Omega \in \mathbb{R}^{+},
\end{aligned}
$$

We suppose that the functions $V_{\omega}$ and $V_{\omega \omega^{\prime}}$ are endowed with some analytic properties that we will specify below equation (6). The generalized right (left) eigenvectors $|1\rangle$ and $|\omega\rangle(\langle 1|$ and $\langle\omega|)$ of $\stackrel{0}{H}$ form a complete biorthogonal set that we can use to expand any vector state $|\Phi\rangle$, i.e.

$$
\langle 1 \mid 1\rangle=1, \quad\left\langle\omega \mid \omega^{\prime}\right\rangle=\delta\left(\omega-\omega^{\prime}\right), \quad\langle 1 \mid \omega\rangle=\langle\omega \mid 1\rangle=0, \quad|\Phi\rangle=|1\rangle\langle 1 \mid \Phi\rangle+\int_{0}^{\infty} d \omega|\omega\rangle\langle\omega \mid \Phi\rangle .
$$

The amplitude of a state vector $\Phi$, observed in the state vector $\Psi$, is given by

$$
\langle\Psi \mid \Phi\rangle=\langle\Psi \mid 1\rangle\langle 1 \mid \Phi\rangle+\int_{0}^{\infty} d \omega\langle\Psi \mid \omega\rangle\langle\omega \mid \Phi\rangle,
$$

and therefore

$$
\langle\Psi \mid \Phi\rangle=\langle\Psi|I| \Phi\rangle, \quad I \doteq|1\rangle\left\langle 1\left|+\int_{0}^{\infty} d \omega\right| \omega\right\rangle\langle\omega|
$$

If we try to construct a complete biorthogonal set of eigenvectors of the Hamiltonian $H=\stackrel{0}{H}+\stackrel{1}{H}$, depending analytically on the small interaction parameter which we assume included in $\stackrel{1}{H}$, the standard methods of perturbation 
theory are not applicable, because the superposition of the discrete and continuous part of the spectrum of $H^{0}$ will produce non defined terms in the perturbation expansion. However, this problem can be avoided if we are satisfied to obtain information about terms of the form $\langle\Psi \mid \Phi\rangle,\langle\Psi|H| \Phi\rangle$ or $\langle\Psi|\exp (-i H t)| \Phi\rangle$, where $\Phi$ is a vector for which $\varphi(\omega)=\langle\omega \mid \Phi\rangle$ has a well defined analytic extension $\varphi(z)$ to the lower half plane, and $\Psi$ is a vector for which $\psi(\omega)=\langle\omega \mid \Psi\rangle$ has a well defined analytic extension $\psi(z)$ to the upper half plane. In this case the functionals $\langle z|$ and $|z\rangle$ are defined through the analytic expressions [4] [5]:

$$
\langle z \mid \Phi\rangle \doteq \varphi(z), \quad\langle\Psi \mid z\rangle \doteq \overline{\psi(\bar{z})}
$$

Then, the Cauchy theorem can be used in equation (3) to write

$$
\langle\Psi \mid \Phi\rangle=\langle\Psi \mid 1\rangle\langle 1 \mid \Phi\rangle+\int_{0}^{\infty} d \omega\langle\Psi \mid \omega\rangle\langle\omega \mid \Phi\rangle=\langle\Psi \mid 1\rangle\langle 1 \mid \Phi\rangle+\int_{\Gamma} d z\langle\Psi \mid z\rangle\langle z \mid \Phi\rangle,
$$

where $\Gamma$ is the curve in the lower complex half plane indicated in Fig. 1 . Therefore, for the states $\Phi$ and observables $\Psi$ endowed with the analyticity properties mentioned above, we can define the new "identity operator" $I_{\Gamma}$

$$
I_{\Gamma} \doteq|1\rangle\left\langle 1\left|+\int_{\Gamma} d z\right| z\right\rangle\langle z|, \quad\langle\Psi \mid \Phi\rangle=\left\langle\Psi\left|I_{\Gamma}\right| \Phi\right\rangle .
$$

The functionals $|1\rangle,\langle 1|| z$,$\rangle and \langle z|$ satisfy

$$
\langle 1 \mid 1\rangle=1, \quad\left\langle z \mid z^{\prime}\right\rangle=\delta_{\Gamma}\left(z-z^{\prime}\right), \quad\langle 1 \mid z\rangle=\langle z \mid 1\rangle=0
$$

where $\delta_{\Gamma}\left(z-z^{\prime}\right)$ is the $\delta$ distribution defined on the curve $\Gamma\left(\int_{\Gamma} d z^{\prime} \delta_{\Gamma}\left(z-z^{\prime}\right) f\left(z^{\prime}\right)=f(z)\right)$.

We can also define $H_{\Gamma}=\stackrel{0}{H} \Gamma+\stackrel{1}{H}{ }_{\Gamma}$, where

$$
\stackrel{0}{H} \Gamma_{\Gamma} \doteq \Omega|1\rangle\left\langle 1\left|+\int_{\Gamma} d z z\right| z\right\rangle\left\langle z\left|, \quad \stackrel{1}{H}_{\Gamma} \doteq \int_{\Gamma} d z V_{z}\right| z\right\rangle\left\langle 1\left|+\int_{\Gamma} d z \overline{V_{\bar{z}}}\right| 1\right\rangle\left\langle z\left|+\int_{\Gamma} d z \int_{\Gamma} d z^{\prime} V_{z z^{\prime}}\right| z\right\rangle\left\langle z^{\prime}\right|,
$$

where $V_{z}, \overline{V_{\bar{z}}}$ and $V_{z z^{\prime}}$ are the analytic extensions to the lower half plane of $V_{\omega}, \overline{V_{\omega}}$ and $V_{\omega \omega^{\prime}}$. Therefore we postulate that these functions can be analytically extended at least up to the curve $\Gamma$. The operators $\stackrel{0}{H}{ }_{\Gamma}$ and $\stackrel{1}{H}{ }_{\Gamma}$ verify $\langle\Psi|\stackrel{0}{H}| \Phi\rangle=\left\langle\Psi \stackrel{0}{H}_{\Gamma} \mid \Phi\right\rangle$ and $\langle\Psi|\stackrel{1}{H}| \Phi\rangle=\langle\Psi|\stackrel{1}{H} \Gamma| \Phi\rangle$ for the restricted class of vectors defined above, and therefore $H_{\Gamma}$ can be considered as the generator of the time evolution. Being $\Omega$ a real number, there is no superposition between the discrete and the continuous part of the spectrum of $\stackrel{0}{H}{ }_{\Gamma}$.

We can now proceed with a perturbation approach to the right eigenvalue problem

$$
H_{\Gamma}|\Phi\rangle=\lambda|\Phi\rangle
$$

By assuming the expansion

$$
|\Phi\rangle=|\stackrel{0}{\Phi}\rangle+|\stackrel{1}{\Phi}\rangle+|\stackrel{2}{\Phi}\rangle+\cdots \cdots, \quad \lambda \stackrel{0}{=}+\stackrel{1}{\lambda}+\stackrel{2}{\lambda}+\cdots \cdots
$$

with respect to the small interaction parameter that we suppose contained in $\stackrel{1}{H_{\Gamma}}$, we obtain order by order the set of equations

$$
\begin{aligned}
& \left(\begin{array}{l}
0 \\
\lambda
\end{array} \stackrel{0}{H_{\Gamma}}\right)|\stackrel{0}{\Phi}\rangle=0 \\
& \left(\begin{array}{l}
0 \\
\lambda
\end{array}-\stackrel{0}{H} \Gamma\right)|\stackrel{1}{\Phi}\rangle=\left(\stackrel{1}{H} \Gamma-\frac{1}{\lambda}\right)|\stackrel{0}{\Phi}\rangle
\end{aligned}
$$

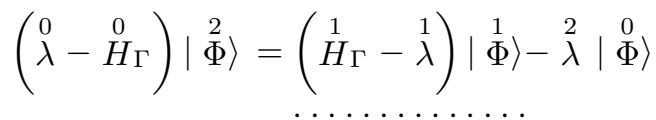

$$
\begin{aligned}
& \left(\begin{array}{l}
0 \\
\lambda
\end{array}-\stackrel{0}{H} \Gamma\right)|\stackrel{n}{\Phi}\rangle=\left(\stackrel{1}{H} \Gamma-\frac{1}{\lambda}\right)|\stackrel{n-1}{\Phi}\rangle-\stackrel{2}{\lambda}|\stackrel{n-2}{\Phi}\rangle-\cdots-\stackrel{n}{\lambda}|\stackrel{0}{\Phi}\rangle
\end{aligned}
$$


Analogously, for the left eigenvalue problem we have

$$
\langle\Psi| H_{\Gamma}=\lambda\langle\Psi|, \quad\langle\Psi|=\langle\stackrel{0}{\Psi}|+\langle\stackrel{1}{\Psi}|+\langle\stackrel{2}{\Psi}|+\cdots, \quad \lambda=\stackrel{0}{\lambda}+\stackrel{1}{\lambda}+\stackrel{2}{\lambda}+\cdots \cdot,
$$

and we obtain

$$
\begin{aligned}
& \langle\stackrel{0}{\Psi}|\left(\begin{array}{l}
0 \\
\lambda
\end{array}-\stackrel{0}{H} \Gamma\right)=0 \\
& \langle\stackrel{1}{\Psi}|\left(\begin{array}{l}
0 \\
\lambda
\end{array}-\stackrel{0}{H} \Gamma\right)=\langle\stackrel{0}{\Psi}|\left(\stackrel{1}{H_{\Gamma}}-\stackrel{1}{\lambda}\right) \\
& \langle\stackrel{2}{\Psi}|\left(\stackrel{0}{\lambda}-\stackrel{0}{H_{\Gamma}}\right)=\langle\stackrel{1}{\Psi}|\left(\stackrel{1}{H_{\Gamma}}-\stackrel{1}{\lambda}\right)-\langle\stackrel{0}{\Psi}| \stackrel{2}{\lambda} \\
& \langle\stackrel{n}{\Psi}|\left(\begin{array}{l}
0 \\
\lambda
\end{array}-\stackrel{0}{H} \Gamma\right)=\langle\stackrel{n-1}{\Psi}|\left(\stackrel{1}{H_{\Gamma}}-\stackrel{1}{\lambda}\right)-\langle\stackrel{n-2}{\Psi}| \stackrel{2}{\lambda}-\cdots-\langle\stackrel{0}{\Psi}| \stackrel{n}{\lambda}
\end{aligned}
$$

It is convenient to define the projectors

$$
P_{d} \doteq|1\rangle\left\langle 1\left|, \quad P_{\Gamma} \doteq \int_{\Gamma} d z\right| z\right\rangle\left\langle z\left|, \quad P_{z} \doteq\right| z\right\rangle\langle z|,
$$

endowed with the following properties

$$
\begin{array}{ccc}
P_{d}+P_{\Gamma} & =I_{\Gamma}, \quad P_{d} P_{d}=P_{d}, \quad P_{\Gamma} P_{\Gamma}=P_{\Gamma}, \quad P_{z} P_{z^{\prime}}=\delta_{\Gamma}\left(z-z^{\prime}\right) P_{z}, \quad P_{d} P_{\Gamma}=P_{\Gamma} P_{d}=0 \\
\stackrel{0}{H} & \stackrel{0}{H}{ }_{\Gamma} P_{d}=\Omega P_{d}, \quad P_{\Gamma} \stackrel{0}{H} \stackrel{0}{H}_{\Gamma} P_{\Gamma}, \quad P_{z} \stackrel{0}{H}_{\Gamma}=\stackrel{0}{H}_{\Gamma} P_{z}=z P_{z} .
\end{array}
$$

With all these equations in hand we can begin our computations.

\section{A. The discrete spectrum.}

Let us start computing the eigenvalues and eigenvectors of $H_{\Gamma}$ corresponding to the unperturbed eigenvalue $\Omega$, so we take $|\stackrel{0}{\Phi}\rangle=|1\rangle$ and $\stackrel{0}{\lambda}=\Omega$. With this choice equation (7) is verified. Then equation (8) gives

$$
\int_{\Gamma} d z|z\rangle(\Omega-z)\langle z \mid \stackrel{1}{\Phi}\rangle=\int_{\Gamma} d z|z\rangle\langle z|\stackrel{1}{H} \Gamma| 1\rangle-\stackrel{1}{\lambda}|1\rangle
$$

and therefore

$$
\stackrel{1}{\lambda}=0, \quad P_{\Gamma}|\stackrel{1}{\Phi}\rangle=\frac{1}{\left(\Omega-\stackrel{0}{H}_{\Gamma}\right)} P_{\Gamma} \stackrel{1}{H}{ }_{\Gamma}|1\rangle .
$$

Equation (9), gives

$$
\int_{\Gamma} d z|z\rangle(\Omega-z)\langle z \mid \stackrel{2}{\Phi}\rangle=\stackrel{1}{=}{ }_{\Gamma}|\stackrel{1}{\Phi}\rangle-\stackrel{2}{\lambda}|1\rangle
$$

and therefore

$$
\stackrel{2}{\lambda}=\langle 1|\stackrel{1}{H} \Gamma| \stackrel{1}{\Phi}\rangle, \quad P_{\Gamma}|\stackrel{2}{\Phi}\rangle=\frac{1}{(\Omega-\stackrel{0}{H} \Gamma)} P_{\Gamma} \stackrel{1}{H} \Gamma|\stackrel{1}{\Phi}\rangle .
$$

For $|\stackrel{0}{\Phi}\rangle=|1\rangle$ and $\stackrel{0}{\lambda}=\Omega$, equations (7)-(10) give no information about $\langle 1 \mid \stackrel{n}{\Phi}\rangle(n=1,2, \ldots)$. Thus, we impose the condition $\langle 1 \mid \stackrel{n}{\Phi}\rangle=0(n=1,2, \ldots)$, namely the usual choice in quantum perturbation theory. This choice will fix the normalization of the eigenvector. In summary, up to second order, we obtain the right eigenvector and eigenvalue 


$$
\begin{aligned}
\left|\Phi_{\Omega}\right\rangle & =|1\rangle+\left(\frac{1}{(\Omega-\stackrel{0}{H} \Gamma)} P_{\Gamma} \stackrel{1}{H}{ }_{\Gamma}\right)|1\rangle+\left(\frac{1}{\left(\Omega-\stackrel{0}{H}{ }_{\Gamma}\right)} P_{\Gamma} \stackrel{1}{H_{\Gamma}}\right)^{2}|1\rangle, \\
\lambda_{\Omega} & =\Omega+\left\langle 1\left|\stackrel{1}{H_{\Gamma}} \frac{1}{\left(\Omega-\stackrel{0}{H}_{\Gamma}\right)} P_{\Gamma} \stackrel{1}{H_{\Gamma}}\right| 1\right\rangle .
\end{aligned}
$$

Using the expressions for $\stackrel{0}{H} \Gamma$ and $\stackrel{1}{H} \Gamma$ given in equations (6), we obtain

$$
\lambda_{\Omega}=\Omega+\int_{\Gamma} d z \frac{V_{z} \overline{V_{\bar{z}}}}{\Omega-z} .
$$

If $V_{z}$ and $\overline{V_{\bar{z}}}$ are assumed to be analytic functions of $z$ in the lower half plane, the integral over $\Gamma$ can be transformed into an integral over $\mathbb{R}^{+}$:

$$
\begin{aligned}
\lambda_{\Omega} & =\Omega+\int_{0}^{\infty} d \omega \frac{V_{\omega} \overline{V_{\omega}}}{\Omega-\omega+i 0}=\Omega+\int_{0}^{\infty} d \omega\left\{-i \pi \delta(\omega-\Omega)-\mathcal{P}\left(\frac{1}{\omega-\Omega}\right)\right\}\left|V_{\omega}\right|^{2}= \\
& =\Omega-\int_{0}^{\infty} d \omega \mathcal{P}\left(\frac{1}{\omega-\Omega}\right)\left|V_{\omega}\right|^{2}-i \pi\left|V_{\Omega}\right|^{2},
\end{aligned}
$$

where we see that the perturbation of the eigenvalue $\Omega>0$ gives, up to second order, a complex eigenvalue $\lambda_{\Omega}$ with negative imaginary part (see Fig.1). Notice that the imaginary part would not appear if $\Omega<0$.

Starting from $\langle\stackrel{0}{\Psi}|=\langle 1|$ and $\stackrel{0}{\lambda}=\Omega$, equations (11)-(14) and the imposed conditions $\stackrel{n}{\Psi}|1\rangle=0(n=1,2, \ldots)$ provide a well defined algorithm to obtain the left eigenvector. E.g. up to second order we obtain

$$
\left\langle\Psi_{\Omega}\right|=\langle 1|+\langle 1|\left(\stackrel{1}{H}_{\Gamma} P_{\Gamma} \frac{1}{\left(\Omega-\stackrel{0}{H}_{\Gamma}\right)}\right)+\langle 1|\left(\stackrel{1}{H}_{\Gamma} P_{\Gamma} \frac{1}{\left(\Omega-\stackrel{0}{H}_{\Gamma}\right)}\right)^{2},
$$

with the same eigenvalue $\lambda_{\Omega}$. As we are searching for a complete biorthogonal system, it is necessary at every step to define the normalized eigenvectors as

$$
\left|f_{\Omega}\right\rangle \doteq\left|\Phi_{\Omega}\right\rangle / \sqrt{\left\langle\Psi_{\Omega} \mid \Phi_{\Omega}\right\rangle}, \quad\left\langle\widetilde{f}_{\Omega}\right| \doteq\left\langle\Psi_{\Omega}\right| / \sqrt{\left\langle\Psi_{\Omega} \mid \Phi_{\Omega}\right\rangle},
$$

which satisfy the condition $\left\langle\widetilde{f}_{\Omega} \mid f_{\Omega}\right\rangle=1$.

\section{B. The continuous spectrum.}

Let us now compute the eigenvalues and eigenvectors of $H_{\Gamma}$ corresponding to the continuous spectrum of $\stackrel{0}{H_{\Gamma}}$. So we now consider equations (7)-(10) with $|\stackrel{0}{\Phi}\rangle=|u\rangle$ and $\stackrel{0}{\lambda}=u(u \in \Gamma)$. Equation (7) is satisfied, and equation (8) gives

$$
\int_{\Gamma} d z|z\rangle(u-z)\langle z \mid \stackrel{1}{\Phi}\rangle+(u-\Omega)|1\rangle\langle 1 \mid \stackrel{1}{\Phi}\rangle=\int_{\Gamma} d z|z\rangle\left\langle z\left|\stackrel{1}{H_{\Gamma}}\right| u\right\rangle+|1\rangle\left\langle 1\left|\stackrel{1}{H}{ }_{\Gamma}\right| u\right\rangle-\stackrel{1}{\lambda} \int_{\Gamma} d z|z\rangle \delta_{\Gamma}(z-u),
$$

or equivalently

$$
(u-z)\langle z \mid \stackrel{1}{\Phi}\rangle=\langle z|\stackrel{1}{H} \Gamma| u\rangle-\stackrel{1}{\lambda} \delta_{\Gamma}(z-u), \quad(u-\Omega)\langle 1 \mid \stackrel{1}{\Phi}\rangle=\langle 1|\stackrel{1}{H} \Gamma| u\rangle
$$

If we assume that $V_{z u}$ is an analytic function of the two variables $z$ and $u$, the term $\left\langle z\left|\stackrel{1}{H_{\Gamma}}\right| u\right\rangle=V_{z u}$ does not include a term proportional to $\delta_{\Gamma}(z-u)$, and therefore equations (21) give

$$
\stackrel{1}{\lambda}=0 \quad P_{\Gamma}|\stackrel{1}{\Phi}\rangle=\frac{1}{(u+i 0-\stackrel{0}{H} \Gamma)} P_{\Gamma} \stackrel{1}{H}{ }_{\Gamma}|u\rangle, \quad P_{d}|\stackrel{1}{\Phi}\rangle=\frac{1}{(u-\stackrel{0}{H} \Gamma)} P_{d} \stackrel{1}{H}{ }_{\Gamma}|u\rangle
$$

Equation (9) gives 


$$
\int_{\Gamma} d z|z\rangle(u-z)\langle z \mid \stackrel{2}{\Phi}\rangle+(u-\Omega)|1\rangle\langle 1 \mid \stackrel{2}{\Phi}\rangle=\int_{\Gamma} d z|z\rangle\left\langle z\left|\stackrel{1}{H} \Gamma_{\Gamma}\right| \stackrel{1}{\Phi}\right\rangle+|1\rangle\langle 1 \mid \stackrel{1}{H} \Gamma \stackrel{1}{\Phi}\rangle-\stackrel{2}{\lambda} \int_{\Gamma} d z|z\rangle \delta_{\Gamma}(z-u),
$$

and therefore

$$
(u-z)\langle z \mid \stackrel{2}{\Phi}\rangle=\left\langle z\left|\stackrel{1}{H}{ }_{\Gamma}\right| \stackrel{1}{\Phi}\right\rangle-\stackrel{2}{\lambda} \delta_{\Gamma}(z-u), \quad(u-\Omega)\langle 1 \mid \stackrel{2}{\Phi}\rangle=\left\langle 1\left|\stackrel{1}{H} \Gamma_{\Gamma}\right| \stackrel{1}{\Phi}\right\rangle .
$$

Using (6) and (22) we can compute

$$
\left\langle z\left|\stackrel{1}{H}_{\Gamma}\right| \stackrel{1}{\Phi}\right\rangle=\int_{\Gamma} d z^{\prime} \frac{V_{z z^{\prime}} V_{z^{\prime} u}}{u+i 0-z^{\prime}}+\frac{V_{z} \overline{V_{\bar{z}}}}{u-\Omega}
$$

which does not include any factor proportional to $\delta_{\Gamma}(z-u)$. Therefore, equations (23) give

$$
\stackrel{2}{\lambda}=0 \quad P_{\Gamma}|\stackrel{2}{\Phi}\rangle=\frac{1}{(u+i 0-\stackrel{0}{H} \Gamma)} P_{\Gamma} \stackrel{1}{H} \Gamma|\stackrel{1}{\Phi}\rangle, \quad P_{d}|\stackrel{2}{\Phi}\rangle=\frac{1}{\left(u-\stackrel{0}{H}{ }_{\Gamma}\right)} P_{d} \stackrel{1}{H} \Gamma_{\Gamma}|\stackrel{1}{\Phi}\rangle
$$

In summary, up to second order, we obtain the right eigenvector

$$
\begin{aligned}
\left|f_{u}\right\rangle= & |u\rangle+\left(\frac{1}{\left(u+i 0-\stackrel{0}{H}_{\Gamma}\right)} P_{\Gamma} \stackrel{1}{H_{\Gamma}}+\frac{1}{\left(u-\stackrel{0}{H}_{\Gamma}\right)} P_{d} \stackrel{1}{H}\right)|u\rangle+ \\
& +\left(\frac{1}{\left(u+i 0-\stackrel{0}{H}{ }_{\Gamma}\right)} P_{\Gamma} \stackrel{1}{H} \Gamma+\frac{1}{\left(u-\stackrel{0}{H}_{\Gamma}\right)} P_{d} \stackrel{1}{H}\right)_{\Gamma}^{2}|u\rangle,
\end{aligned}
$$

with eigenvalue $\lambda_{u}=u$. Notice that we have included the factor $i 0$, namely we make the usual choice of outgoing solutions of scattering theory, to avoid the continuous-continuous resonances.

Equations (11)-(14) with $\langle\stackrel{0}{\Psi}|=\langle u|$ and $\stackrel{0}{\lambda}=u \in \Gamma$ are used to obtain the corresponding left eigenvector. Up to second order we obtain the left eigenvector

$$
\begin{gathered}
\left\langle\widetilde{f}_{u}\right|=\langle u|+\langle u|\left(\stackrel{1}{H_{\Gamma}} P_{\Gamma} \frac{1}{(u-i 0-\stackrel{0}{H} \Gamma)}+\stackrel{1}{H_{\Gamma}} P_{d} \frac{1}{\left(u-\stackrel{0}{H}_{\Gamma}\right)}\right)+ \\
\langle u|\left(\stackrel{1}{H_{\Gamma}} P_{\Gamma} \frac{1}{\left(u-i 0-\stackrel{0}{H}_{\Gamma}\right)}+\stackrel{1}{H} \Gamma P_{d} \frac{1}{\left(u-\stackrel{0}{H}_{\Gamma}\right)}\right)^{2} .
\end{gathered}
$$

It can be easily verified that the vectors given in equations (20)-(25) satisfy, up to second order, the orthogonality relations

$$
\left\langle\tilde{f}_{\Omega} \mid f_{\Omega}\right\rangle=1, \quad\left\langle\tilde{f}_{u} \mid f_{u^{\prime}}\right\rangle=\delta_{\Gamma}\left(u-u^{\prime}\right), \quad\left\langle\tilde{f}_{\Omega} \mid f_{u}\right\rangle=\left\langle\tilde{f}_{u} \mid f_{\Omega}\right\rangle=0
$$

They also verify, up to second order, the "completeness relation"

$$
\langle\Psi \mid \Phi\rangle=\left\langle\Psi\left|I_{\Gamma}\right| \Phi\right\rangle, \quad I_{\Gamma} \doteq|1\rangle\left\langle 1\left|+\int_{\Gamma} d u\right| u\right\rangle\left\langle u|=| f_{\Omega}\right\rangle\left\langle\widetilde{f}_{\Omega}\left|+\int_{\Gamma} d u\right| f_{u}\right\rangle\left\langle\widetilde{f}_{u}\right| .
$$

For the operators $H$ and $H_{\Gamma}$ defined in equations (11) and (6) we also have

$$
\langle\Psi|H| \Phi\rangle=\left\langle\Psi\left|H_{\Gamma}\right| \Phi\right\rangle, \quad H_{\Gamma}=\lambda_{\Omega}\left|f_{\Omega}\right\rangle\left\langle\widetilde{f}_{\Omega}\left|+\int_{\Gamma} d u \lambda_{u}\right| f_{u}\right\rangle\left\langle\widetilde{f}_{u}\right|,
$$

showing that the eigenvalues and eigenvectors obtained above provide the spectral decomposition of $H_{\Gamma}$. It should be stressed that the expressions for $I_{\Gamma}$ and $H_{\Gamma}$ given in equations (27) and (28)are meaningful only when they are used to compute $\left\langle\Psi\left|I_{\Gamma}\right| \Phi\right\rangle$ or $\left\langle\Psi\left|H_{\Gamma}\right| \Phi\right\rangle$ for vectors $\Psi(\Phi)$ for which $\psi(\omega)=\langle\omega \mid \Psi\rangle(\varphi(\omega)=\langle\omega \mid \Phi\rangle)$ have a well defined analytic extension to the complex upper (lower) half plane. 


\section{The time evolution.}

Let us make a first application of the above results. The probability $P_{t}$ of the state vector $\Phi_{t}=e^{-i H t} \Phi_{0}$ at the time $t$ of being measured in the state $\Psi$ is given by

$$
P_{t}=\left|\left\langle\Psi|\exp (-i H t)| \Phi_{0}\right\rangle\right|^{2}=\left|\left\langle\Psi\left|\exp \left(-i H_{\Gamma} t\right)\right| \Phi_{0}\right\rangle\right|^{2},
$$

where

$$
\left\langle\Psi\left|\exp \left(-i H_{\Gamma} t\right)\right| \Phi_{0}\right\rangle=\exp \left(-i \lambda_{\Omega} t\right)\left\langle\Psi \mid f_{\Omega}\right\rangle\left\langle\tilde{f}_{\Omega} \mid \Phi_{0}\right\rangle+\int_{\Gamma} d u \exp (-i u t)\left\langle\Psi \mid f_{u}\right\rangle\left\langle\tilde{f}_{u} \mid \Phi_{0}\right\rangle
$$

If the exact expressions for the generalized eigenvalues and eigenvectors were known, equations (29) and (30) would give $P_{t}$ exactly. An approximated expression will be obtained if eigenvalues and eigenvectors are given up to some finite order in the perturbation expansion.

Let us consider the survival amplitude of the unstable state $|1\rangle$, given by

$$
\left\langle 1\left|\exp \left(-i H_{\Gamma} t\right)\right| 1\right\rangle=\exp \left(-i \lambda_{\Omega} t\right)\left\langle 1 \mid f_{\Omega}\right\rangle\left\langle\widetilde{f}_{\Omega} \mid 1\right\rangle+\int_{\Gamma} d u \exp (-i u t)\left\langle 1 \mid f_{u}\right\rangle\left\langle\widetilde{f}_{u} \mid 1\right\rangle .
$$

For a small coupling between the discrete and the continuous spectrum, i.e. if in the expression for $\stackrel{1}{H}$ given in equation (11) there is a small multiplicative parameter $\varepsilon$ included in $V_{\omega}$, and using the explicit expressions of the generalized eigenvectors given in sections IIIA and IIIB, we obtain

$$
\left\langle 1 \mid f_{\Omega}\right\rangle\left\langle\widetilde{f}_{\Omega} \mid 1\right\rangle=1+O\left(\varepsilon^{2}\right), \quad\left\langle 1 \mid f_{u}\right\rangle\left\langle\tilde{f}_{u} \mid 1\right\rangle=O\left(\varepsilon^{2}\right)
$$

If $\lambda_{\Omega}$ is computed up to second order from equation (18), we have

$$
|\langle 1|\exp (-i H t)| 1\rangle|^{2} \cong \exp \left(-2 \pi V_{\Omega}^{2} t\right)
$$

This expression is valid if $\varepsilon^{2} \ll 1$ and $\varepsilon^{2} t \leq 1$, i.e. for small coupling between the discrete and the continuous spectrums and for not too large times. This is the standard approximated expression for a decay process which can be found in several text books on quantum mechanics [12]. The deviations from exponential behavior (Zeno and Khalfin effects for shot and long times respectively), are not shown in this approximation. Concerning Zeno effect, notice that the time derivative for $t=0$ of expression (32), is of order $\varepsilon^{2}$. If we compute the survival probability keeping the second order terms coming from equations (31), we can obtain the Zeno effect, because the initial time derivative at $t=0$ turns out to be of order $\varepsilon^{4}$.

\section{Tunneling through a barrier.}

We now consider some particular models. Let us first consider the one dimensional rectangular well problem with the following Hamiltonian, given in coordinate representation

$$
\stackrel{0^{\prime}}{H}=-\frac{\hbar^{2}}{2 \mu} \frac{d^{2}}{d x^{2}}+\stackrel{0}{V}(x)
$$

where

$$
\stackrel{0}{V}(x)=\begin{array}{ll}
V_{0} & |x|>a \\
0 & |x|<a
\end{array}
$$

The spectral decomposition of $\stackrel{0}{H}^{\prime}$ is well known. The discrete part of the spectrum has eigenvalues $E_{j}$ between 0 and $V_{0}$. In coordinate representation, the corresponding normalized eigenvectors $\langle x \mid j\rangle$ are of the form $\exp \left(\mp \sqrt{\frac{2 \mu}{\hbar^{2}}\left(V_{0}-E_{j}\right)} x\right)$, the minus (plus) sign corresponding to $x>a(x<-a)$. Therefore the vector states $\langle x \mid j\rangle$ are concentrated inside the rectangular well. We are going to consider the simple case in which the parameters $a$ and $V_{0}$ verify the condition $a \sqrt{\frac{2 \mu V_{0}}{\hbar^{2}}}<\frac{\pi}{2}$, where there is a single discrete eigenvalue $E_{1}$, and the corresponding 
eigenvector $\langle x \mid 1\rangle$ is an even function of the coordinate $x$ [13]. There is also a continuous spectrum with generalized eigenvalues $E_{k}=\frac{\hbar^{2}}{2 \mu} k^{2}+V_{0}(-\infty<k<+\infty)$. The generalized eigenvectors $|k\rangle$ can be obtained as the solutions of the Lipmann-Schwinger equation. The vectors $|k\rangle$ and $|1\rangle$ form an orthonormal basis

$$
\left\langle k \mid k^{\prime}\right\rangle=\delta\left(k-k^{\prime}\right), \quad\langle 1 \mid 1\rangle=1, \quad\langle 1 \mid k\rangle=\langle k \mid 1\rangle=0, \quad I=|1\rangle\left\langle 1\left|+\int d k\right| k\right\rangle\langle k| .
$$

We can now add to $\stackrel{0}{H}^{\prime}$ the potential

$$
\stackrel{1}{\prime}^{\prime}(x)=\begin{array}{ll}
0 & |x|<b \\
-V_{1} & |x|>b
\end{array}
$$

with $b \gg a$ and $0<V_{1}<V_{0}$. Thus, a barrier of height $V_{0}-V_{1}$ and length $b-a$ appears. The potential $\stackrel{1}{V}^{\prime}$ can be written in terms of the basis given in (35)

$$
\stackrel{1^{\prime}}{V}=|1\rangle V_{11}\left\langle 1\left|+\int d k^{\prime}\right| 1\right\rangle V_{1 k^{\prime}}\left\langle k^{\prime}\left|+\int d k V_{k 1}\right| k\right\rangle\left\langle 1\left|+\int d k \int d k^{\prime}\right| k\right\rangle \widetilde{V}_{k k^{\prime}}\left\langle k^{\prime}\right|,
$$

where

$$
\begin{gathered}
V_{11}=-V_{1}\left[\int_{-\infty}^{-b} d x\langle 1 \mid x\rangle\langle x \mid 1\rangle+\int_{+b}^{+\infty} d x\langle 1 \mid x\rangle\langle x \mid 1\rangle\right], \\
V_{1 k}=\overline{V_{k 1}}=-V_{1}\left[\int_{-\infty}^{-b} d x\langle 1 \mid x\rangle\langle x \mid k\rangle+\int_{+b}^{+\infty} d x\langle 1 \mid x\rangle\langle x \mid k\rangle\right], \\
\widetilde{V}_{k k^{\prime}}=-V_{1} \delta\left(k-k^{\prime}\right)+V_{k k^{\prime}}, \quad V_{k k^{\prime}}=V_{1} \int_{-b}^{+b} d x\langle k \mid x\rangle\left\langle x \mid k^{\prime}\right\rangle .
\end{gathered}
$$

Due to the exponential decrease of $\langle x \mid 1\rangle$ when $x \rightarrow \pm \infty$, together with the assumption $b \gg a$, it can be expected that $V_{11}$ and $V_{1 k}$ be small.

The total Hamiltonian $H=\stackrel{0^{\prime}}{H}+\stackrel{1}{V}^{\prime}$ can be also given by $H \stackrel{0}{H}+\stackrel{1}{H}$ where

$$
\begin{aligned}
& \stackrel{0}{H}=\left(E_{1}+V_{11}\right)|1\rangle\left\langle 1\left|+\int_{-\infty}^{+\infty} d k\left(E_{k}-V_{1}\right)\right| k\right\rangle\langle k|, \\
& \stackrel{1}{H}=\int_{-\infty}^{+\infty} d k^{\prime}|1\rangle V_{1 k^{\prime}}\left\langle k^{\prime}\left|+\int_{-\infty}^{+\infty} d k V_{k 1}\right| k\right\rangle\left\langle 1\left|+\int_{-\infty}^{+\infty} d k \int_{-\infty}^{+\infty} d k^{\prime}\right| k\right\rangle V_{k k^{\prime}}\left\langle k^{\prime}\right| .
\end{aligned}
$$

Our interest is to describe the time evolution of the unstable state $|1\rangle$, which is an even function in coordinate representation. Only the even part of the Hamiltonian is relevant to this process

$$
\begin{aligned}
& \stackrel{0}{H} \text { even }^{=}\left(E_{1}+V_{11}\right)|1\rangle\left\langle 1\left|+\int_{0}^{+\infty} d k\left(E_{k}-V_{1}\right)\right| k_{\text {even }}\right\rangle\left\langle k_{\text {even }}\right|, \\
& \stackrel{1}{H}_{\text {even }}=\int_{0}^{+\infty} d k^{\prime}|1\rangle V_{1 k^{\prime}}\left\langle k_{\text {even }}^{\prime}\left|+\int_{0}^{+\infty} d k V_{k 1}\right| k_{\text {even }}\right\rangle\left\langle 1\left|+\int_{0}^{+\infty} d k \int_{0}^{+\infty} d k^{\prime}\right| k_{\text {even }}\right\rangle V_{k k^{\prime}}\left\langle k_{\text {even }}^{\prime}\right|,
\end{aligned}
$$

where $\left|k_{\text {even }}\right\rangle=\frac{1}{\sqrt{2}}(|k\rangle+|-k\rangle)$. If the integrals over $\mathbb{R}^{+}$are deformed into integrals over a curve $\Gamma$ in the lower complex half plane, and if $V_{11}$ and $V_{1 k}$ are small, we can use the perturbation method of the beginning of this section. The correction to the discrete eigenvalue $\left(E_{1}+V_{11}\right)$ of $\stackrel{0}{H}_{\text {even }}$ due to the interaction is

$$
\begin{aligned}
& \left\langle 1\left|\stackrel{1}{H}_{\text {even }} \frac{1}{E_{1}+V_{11}-\stackrel{0}{H}_{\text {even }}+i 0} \stackrel{1}{H}_{\text {even }}\right| 1\right\rangle \\
= & \int_{0}^{+\infty} \frac{V_{1 k} V_{k 1}}{\left(E_{1}+V_{11}\right)-\left(\frac{\hbar^{2} k^{2}}{2 \mu}+V_{0}-V_{1}\right)+i 0} d k= \\
= & -i \pi \int_{0}^{+\infty} V_{1 k} V_{k 1} \delta\left[\left(E_{1}+V_{11}\right)-\left(\frac{\hbar^{2} k^{2}}{2 \mu}+V_{0}-V_{1}\right)\right] d k-\int_{0}^{+\infty} V_{1 k} V_{k 1} \mathcal{P}\left[\frac{1}{\left(E_{1}+V_{11}\right)-\left(\frac{\hbar^{2} k^{2}}{2 \mu}+V_{0}-V_{1}\right)}\right] d k .
\end{aligned}
$$


If $\left(E_{1}+V_{11}\right)>\left(V_{0}-V_{1}\right)$ the corrected eigenvalue has an imaginary part $-i \frac{\pi \mu}{\hbar^{2} \tilde{k}} V_{1 \widetilde{k}} V_{\widetilde{k} 1}$, where

$$
\widetilde{k}=\sqrt{\frac{2 \mu}{\hbar^{2}}\left[\left(E_{1}+V_{11}\right)-\left(V_{0}-V_{1}\right)\right]}
$$

and the survival probability of the unstable state $|1\rangle$ is approximately given by

$$
|\langle 1|\exp (-i H t)| 1\rangle|^{2} \cong \exp \left(-\frac{2 \pi \mu}{\hbar^{2} \widetilde{k}} V_{1 \widetilde{k}} V_{\widetilde{k} 1}\right)
$$

\section{E. Friedrichs model.}

We will finally check our method with the well known solvable Friedrichs model. This model can be obtained as a special case of equation (11), with $V_{\omega \omega^{\prime}}=0$. The Hamiltonian is $H=\stackrel{0}{H}+\stackrel{1}{H}$, where

$$
\stackrel{0}{H}=\Omega|1\rangle\left\langle 1\left|+\int_{0}^{\infty} d \omega \omega\right| \omega\right\rangle\langle\omega|, \quad \stackrel{1}{H}=\int_{0}^{\infty} d \omega\left\{V_{\omega}|\omega\rangle\left\langle 1\left|+\overline{V_{\omega}}\right| 1\right\rangle\langle\omega|\right\}, \quad \Omega \in \mathbb{R}^{+}
$$

As we considered in the previous section, the variable $\omega \in \mathbb{R}^{+}$can be transformed into a variable $z \in \Gamma$, being $\Gamma$ the curve in the lower complex half plane shown in Fig.1. The corresponding Hamiltonian $H_{\Gamma}$ is given by

$$
H_{\Gamma}=\stackrel{0}{H} \Gamma+\stackrel{1}{H} \Gamma, \quad \stackrel{0}{H} \Gamma=\Omega|1\rangle\left\langle 1\left|+\int_{\Gamma} d z z\right| z\right\rangle\left\langle z\left|, \quad \stackrel{1}{H_{\Gamma}}=\int_{\Gamma} d z V_{z}\right| z\right\rangle\left\langle 1\left|+\int_{\Gamma} d z \overline{V_{\bar{z}}}\right| 1\right\rangle\langle z|,
$$

where $V_{z}$ and $\overline{V_{\bar{z}}}$ are the analytic extensions of $V_{\omega}$ and $\overline{V_{\omega}}$.

It is possible to obtain exact solutions for the right and left eigenvalue problems $H_{\Gamma}|\Phi\rangle=\lambda|\Phi\rangle$ and $\langle\Psi| H_{\Gamma}=\lambda\langle\Psi|$, where $|\Phi\rangle=|1\rangle\langle 1 \mid \Phi\rangle+\int_{\Gamma} d z z|z\rangle\langle z \mid \Phi\rangle$ and $\langle\Psi|=\langle\Psi \mid 1\rangle\langle 1|+\int_{\Gamma} d z\langle\Psi \mid z\rangle\langle z|$. If for the sake of simplicity

$$
\eta(\lambda) \doteq \lambda-\Omega-\int_{\Gamma} d z \frac{V_{z} \overline{V_{\bar{z}}}}{\lambda-z}
$$

verifies $\eta\left(\lambda_{\Omega}\right)=0$ for just a single complex number $\lambda_{\Omega}$ located in the region of the complex plane between $\mathbb{R}^{+}$and the curve $\Gamma$, the following spectral decomposition is obtained [1]

$$
H_{\Gamma}=\lambda_{\Omega}\left|f_{\Omega}\right\rangle\left\langle\widetilde{f}_{\Omega}\left|+\int_{\Gamma} d u u\right| f_{u}\right\rangle\left\langle\widetilde{f}_{u}\right|
$$

where

$$
\begin{aligned}
& \left|f_{\Omega}\right\rangle=\frac{1}{\sqrt{\eta^{\prime}\left(\lambda_{\Omega}\right)}}\left\{|1\rangle+\int_{\Gamma} d z \frac{V_{z}}{\lambda_{\Omega}-z}|z\rangle\right\} \\
& \left\langle\widetilde{f}_{\Omega}\right|=\frac{1}{\sqrt{\eta^{\prime}\left(\lambda_{\Omega}\right)}}\left\{\langle 1|+\int_{\Gamma} d z \frac{\overline{V_{\bar{z}}}}{\lambda_{\Omega}-z}\langle z|\right\} \\
& \left|f_{u}\right\rangle=|u\rangle+\frac{\overline{V_{\bar{u}}}}{\eta(u+i \varepsilon)}\left\{|1\rangle+\int_{\Gamma} d z \frac{V_{z}}{(u+i 0)-z}|z\rangle\right\} \\
& \left\langle\widetilde{f}_{u}\right|=\langle u|+\frac{V_{u}}{\eta(u-i 0)}\left\{\langle 1|+\int_{\Gamma} d z \frac{\overline{V_{\bar{z}}}}{(u-i 0)-z}\langle z|\right\}, \quad u \in \Gamma .
\end{aligned}
$$

These generalized eigenvectors form a complete biorthogonal system [1, in the sense given in equations (26) and (27).

Using equations (17)-(20) we can obtain the approximated eigenvectors

$$
\begin{aligned}
& \left|f_{\Omega}\right\rangle \cong|1\rangle+\int_{\Gamma} d z \frac{V_{z}}{\Omega-z}|z\rangle-\frac{1}{2} \int_{\Gamma} d z \frac{V_{z} \overline{V_{\bar{z}}}}{(\Omega-z)^{2}}|1\rangle \\
& \left\langle\widetilde{f}_{\Omega}\right| \cong\langle 1|+\int_{\Gamma} d z \frac{\overline{V_{\bar{z}}}}{\Omega-z}\langle z|-\frac{1}{2} \int_{\Gamma} d z \frac{\overline{V_{\bar{z}}} V_{z}}{(\Omega-z)^{2}}\langle 1|,
\end{aligned}
$$


with the eigenvalue

$$
\lambda_{\Omega} \cong \Omega+\int_{\Gamma} d z \frac{V_{z} \overline{V_{\bar{z}}}}{\Omega-z}=\Omega-\int_{0}^{\infty} d \omega \mathcal{P} \frac{1}{\omega-\Omega}\left|V_{\omega}\right|^{2}-i \pi\left|V_{\Omega}\right|^{2} .
$$

Using equations (24) and (25) we also obtain, up to second order

$$
\begin{aligned}
& \left|f_{u}\right\rangle \cong|u\rangle+\frac{\overline{V_{\bar{u}}}}{u-\Omega}|1\rangle+\frac{\overline{V_{\bar{u}}}}{u-\Omega} \int_{\Gamma} d z \frac{V_{z}}{(u-z+i \varepsilon)}|z\rangle \\
& \left\langle\widetilde{f}_{u}\right| \cong\langle u|+\frac{V_{u}}{u-\Omega}\langle 1|+\frac{V_{u}}{u-\Omega} \int_{\Gamma} d z \frac{\overline{V_{\bar{z}}}}{(u-z-i \varepsilon)}\langle z|,
\end{aligned}
$$

with eigenvalue $\lambda_{u}=u(u \in \Gamma)$.

In order to compare these results with the approximate eigenvalues and eigenvectors obtained in equations (41)-(43), we need an expansion of the exact expressions (40) in powers of the interaction parameter. We can use equation (39) to obtain the proper eigenvalue $\lambda_{\Omega}$, satisfying $\eta\left(\lambda_{\Omega}\right)=0$, from the iterative equation

$$
\stackrel{n+1}{\lambda_{\Omega}}=\Omega+\int_{\Gamma} d z \frac{V_{z} \overline{V_{\bar{z}}}}{\left(\lambda_{\Omega}-z\right)}, \quad, n=0,1,2, \ldots, \quad \stackrel{0}{\lambda}=\Omega .
$$

For small values of the interaction parameter, the secuence $\lambda_{\Omega}^{n}$ converge to a single solution $\lambda_{\Omega}$ of $\eta\left(\lambda_{\Omega}\right)=0$. Up to second order we have

$$
\lambda_{\Omega} \cong \Omega+\int_{\Gamma} d z \frac{V_{z} \overline{V_{\bar{z}}}}{\Omega-z} .
$$

Up to second order in the interaction parameter, we also have

$$
\frac{1}{\sqrt{\eta^{\prime}\left(\lambda_{\Omega}\right)}} \cong 1-\frac{1}{2} \int_{\Gamma} d z \frac{V_{z} \overline{V_{\bar{z}}}}{(\Omega-z)^{2}}, \quad \frac{1}{\lambda_{\Omega}-z}=\frac{1}{\Omega-z}+O\left(V^{2}\right), \quad \frac{1}{\eta(u \pm i \varepsilon)}=\frac{1}{u-\Omega}+O\left(V^{2}\right)
$$

Replacing (44) and (45) in (40) we obtain the approximated expressions (41)-(43), so our approximated algorithm reproduces the results of the solvable model.

\section{GENERALIZED STATES AND SINGULAR OBSERVABLES.}

Up to now we have only considered pure states and observables which can be represented by projectors constructed with normalized vectors. The extension of the formalism to mixed states, represented by density operators of the form $\widehat{\rho}=\sum_{j} p_{j}\left|\Phi_{j}\right\rangle\left\langle\Phi_{j}\right|\left(\left\langle\Phi_{j} \mid \Phi_{j}\right\rangle=1, p_{j} \geq 0, \sum_{j} p_{j}=1\right)$ and observables of the same form $\left(\widehat{O}=\sum_{j} O_{j}\left|\Psi_{j}\right\rangle\left\langle\Psi_{j}\right|\right)$ is straightforward. However, singular observables as $\int_{\omega_{1}}^{\omega_{2}} d \omega|\omega\rangle\langle\omega|$, which are not operators of the form just mentioned, normally appear, and they can not be considered in the framework of the perturbation method presented in the previous section or its straightforward generalization. In this case, it is possible to consider the states as functionals acting on the set of observables represented by operators including singular terms [9] [6] [7]. These generalized states are also useful to study the approach to equilibrium in the thermodynamic limit $[\overline{7}]$. The corresponding perturbation method is therefore a non trivial generalization of the one in section II. This generalization will be the subject of this section and, as we will see, contains new and interesting features.

Let us consider the Friedrichs model, presented in section II. The Hamiltonian is given by equation (37) and we assume, for simplicity, that $V_{\omega}=\overline{V_{\omega}}$.

For this model we are going to consider the class of observables which can be represented by operators of the form

$$
O=O_{1}|1\rangle\left\langle 1\left|+\int_{0}^{\infty} d \omega O_{\omega}\right| \omega\right\rangle\left\langle\omega\left|+\int_{0}^{\infty} d \omega \int_{0}^{\infty} d \omega^{\prime} O_{\omega \omega^{\prime}}\right| \omega\right\rangle\left\langle\omega^{\prime}\left|+\int_{0}^{\infty} d \omega O_{\omega 1}\right| \omega\right\rangle\left\langle 1\left|+\int_{0}^{\infty} d \omega^{\prime} O_{1 \omega^{\prime}}\right| 1\right\rangle\left\langle\omega^{\prime}\right|
$$

where $O_{1}=\overline{O_{1}}, O_{\omega}=\overline{O_{\omega}}, O_{\omega \omega^{\prime}}=\overline{O_{\omega^{\prime} \omega}}$, and $O_{\omega 1}=\overline{O_{1 \omega}}$, being $O_{\omega}, O_{\omega \omega^{\prime}}, O_{\omega 1}$ and $O_{1 \omega^{\prime}}$ regular functions of the variables $\omega$ and $\omega^{\prime}$. The second term of the r.h.s. of the last equation is a singular operator.

In the Heisemberg representation, the time evolution of an observable is given by

\footnotetext{
${ }^{1}$ Superoperators, i.e. operators acting on operators, will be written in blackboard bold types.
} 


$$
-i \frac{d}{d t} O_{t}=\mathbb{L} O_{t}, \quad \mathbb{L} O \doteq[H, O],
$$

where $\mathbb{L}$ is the Liouville-Von Newmann superoperator.

It is convenient to introduce the notation $\mid O) \doteq O$ and to define the generalized operators

$$
\mid 1) \doteq|1\rangle\langle 1|, \quad| \omega) \doteq|\omega\rangle\left\langle\omega|, \quad| \omega \omega^{\prime}\right) \doteq|\omega\rangle\left\langle\omega^{\prime}|, \quad| \omega 1\right) \doteq|\omega\rangle\left\langle 1|, \quad| 1 \omega^{\prime}\right) \doteq|1\rangle\left\langle\omega^{\prime}\right| .
$$

Therefore we can write

$$
\left.\left.\left.\left.\left.\mid O)=O_{1} \mid 1\right)+\int_{0}^{\infty} d \omega O_{\omega} \mid \omega\right)+\int_{0}^{\infty} d \omega \int_{0}^{\infty} d \omega^{\prime} O_{\omega \omega^{\prime}} \mid \omega \omega^{\prime}\right)+\int_{0}^{\infty} d \omega O_{\omega 1} \mid \omega 1\right)+\int_{0}^{\infty} d \omega^{\prime} O_{1 \omega^{\prime}} \mid 1 \omega^{\prime}\right) .
$$

When the states of the system are represented by the usual density operators $\widehat{\rho}=\hat{\rho}^{\dagger}$, the mean value of an observable $O$ is given by $\langle O\rangle_{\widehat{\rho}}=\operatorname{Tr}\left(\widehat{\rho}^{\dagger} O\right)$, and any density operator has the following well known properties

$$
\operatorname{Tr}\left(\widehat{\rho}^{\dagger}\right)=\operatorname{Tr}\left(\widehat{\rho}^{\dagger} I\right)=1, \quad \operatorname{Tr}\left(\widehat{\rho}^{\dagger}[\alpha A+\beta B]=\alpha \operatorname{Tr}\left(\widehat{\rho}^{\dagger} A\right)+\beta \operatorname{Tr}\left(\hat{\rho}^{\dagger} B\right), \quad \operatorname{Tr}\left(\widehat{\rho}^{\dagger} B^{\dagger}\right)=\overline{\operatorname{Tr}\left(\widehat{\rho}^{\dagger} B\right)},\right.
$$

where $I$ is the identity operator $\left.\left(I \doteq|1\rangle\left\langle 1\left|+\int_{0}^{\infty} d \omega\right| \omega\right\rangle\langle\omega|=| 1)+\int_{0}^{\infty} d \omega \mid \omega\right)\right)$.

As we are going to consider the possibility to have more general states than the usual density operators $\widehat{\rho}$, we will consider generalized states as functionals $\rho$ acting on the space of observables defined in equation (48). The action of a state functional $\rho$ on an observable $O$ will be denoted by $(\rho \mid O)$ and it gives the mean value $\langle O\rangle_{\rho}$ of the observable in the state $\rho\left(\langle O\rangle_{\rho} \doteq(\rho \mid O)\right)$. For any state functional $\rho$ we assume the following properties

$$
(\rho \mid I)=1, \quad(\rho \mid \alpha A+\beta B)=\alpha(\rho \mid A)+\beta(\rho \mid B), \quad\left(\rho \mid B^{\dagger}\right)=\overline{(\rho \mid B)},
$$

which are the generalization of the properties for the density operators $\widehat{\rho}$ given in equations (49).

For the class of observables given in equation (48) it is convenient to define the functionals $\left(1 \mid,(\omega),\left(\omega \omega^{\prime} \mid,(\omega 1 \mid\right.\right.$ and $\left(1 \omega^{\prime} \mid\right.$, in such a way that

$$
(1 \mid O)=O_{1}, \quad(\omega \mid O)=O_{\omega}, \quad\left(\omega \omega^{\prime} \mid O\right)=O_{\omega \omega^{\prime}}, \quad(\omega 1 \mid O)=O_{\omega 1}, \quad\left(1 \omega^{\prime} \mid O\right)=O_{1 \omega^{\prime}} .
$$

Using (48), (50) and (51) we obtain

$$
\begin{aligned}
(\rho \mid O)= & (\rho \mid 1)(1 \mid O)+\int_{0}^{\infty} d \omega(\rho \mid \omega)(\omega \mid O)+\int_{0}^{\infty} d \omega \int_{0}^{\infty} d \omega^{\prime}\left(\rho \mid \omega \omega^{\prime}\right)\left(\omega \omega^{\prime} \mid O\right)+ \\
& +\int_{0}^{\infty} d \omega(\rho \mid \omega 1)(\omega 1 \mid O)+\int_{0}^{\infty} d \omega^{\prime}\left(\rho \mid 1 \omega^{\prime}\right)\left(1 \omega^{\prime} \mid O\right) .
\end{aligned}
$$

From this equation we obtain

$$
(\rho \mid O)=(\rho|\mathbb{I}| O),
$$

where $\mathbb{I}$ is the identity superoperator

$$
\mathbb{I} \doteq \mid 1)\left(1\left|+\int_{0}^{\infty} d \omega\right| \omega\right)\left(\omega\left|+\int_{0}^{\infty} d \omega \int_{0}^{\infty} d \omega^{\prime}\right| \omega \omega^{\prime}\right)\left(\omega \omega^{\prime}\left|+\int_{0}^{\infty} d \omega\right| \omega 1\right)\left(\omega 1\left|+\int_{0}^{\infty} d \omega^{\prime}\right| 1 \omega^{\prime}\right)\left(1 \omega^{\prime} \mid .\right.
$$

The generalized state functionals and observables defined in (47) and (51) satisfy the orthogonality conditions

$$
\begin{gathered}
(1 \mid 1)=1, \quad\left(\omega \mid \omega^{\prime}\right)=\delta\left(\omega-\omega^{\prime}\right), \\
\left(\omega \omega^{\prime} \mid \xi \xi^{\prime}\right)=\delta(\omega-\xi) \delta\left(\omega^{\prime}-\xi^{\prime}\right), \quad(\omega 1 \mid \xi 1)=\delta(\omega-\xi), \quad\left(1 \omega^{\prime} \mid 1 \xi^{\prime}\right)=\delta\left(\omega^{\prime}-\xi^{\prime}\right), \\
(1 \mid \omega)=\left(1 \mid \xi \xi^{\prime}\right)=(1 \mid \xi 1)=\left(1 \mid 1 \xi^{\prime}\right)=0, \quad\left(\omega \omega^{\prime} \mid 1\right)=\left(\omega \omega^{\prime} \mid \xi\right)=\left(\omega \omega^{\prime} \mid \xi 1\right)=\left(\omega \omega^{\prime} \mid 1 \xi^{\prime}\right)=0, \\
(\omega 1 \mid 1)=(\omega 1 \mid \xi)=\left(\omega 1 \mid \xi \xi^{\prime}\right)=\left(\omega 1 \mid 1 \xi^{\prime}\right)=0, \quad\left(1 \omega^{\prime} \mid 1\right)=\left(1 \omega^{\prime} \mid \xi\right)=\left(1 \omega^{\prime} \mid \xi \xi^{\prime}\right)=\left(1 \omega^{\prime} \mid \xi 1\right)=0 .
\end{gathered}
$$

Defining the superoperators $\stackrel{0}{\mathbb{L}}$ and $\stackrel{1}{\mathbb{L}}$ by 


$$
\stackrel{0}{\mathbb{L}} O \doteq[\stackrel{0}{H}, O], \quad \stackrel{1}{\mathbb{L}} O \doteq[\stackrel{1}{H}, O], \quad \mathbb{L}=\stackrel{0}{\mathbb{L}}+\stackrel{1}{\mathbb{L}}
$$

and using the generalized operators and functionals defined in equations (47) and (51), we have

$$
\begin{aligned}
\stackrel{0}{\mathbb{L}}= & \left.\int d \omega^{\prime}\left(\Omega-\omega^{\prime}\right) \mid 1 \omega^{\prime}\right)\left(1 \omega^{\prime}\left|+\int d \omega(\omega-\Omega)\right| \omega 1\right)\left(\omega 1\left|+\int d \omega \int d \omega^{\prime}\left(\omega-\omega^{\prime}\right)\right| \omega \omega^{\prime}\right)\left(\omega \omega^{\prime} \mid\right. \\
\stackrel{1}{\mathbb{L}}= & \left.\left.\left.\left.\int d \omega V_{\omega}[\mid \omega 1)-\mid 1 \omega\right)\right]\left(1\left|+\int d \omega V_{\omega}[\mid 1 \omega)-\right| \omega 1\right)\right]\left(\omega\left|+\int d \omega\left[-V_{\omega} \mid 1\right)+\int d \omega^{\prime} V_{\omega^{\prime}}\right| \omega^{\prime} \omega\right)\right](1 \omega \mid+ \\
& \left.\left.\left.+\int d \omega\left[V_{\omega} \mid 1\right)-\int d \omega^{\prime} V_{\omega^{\prime}} \mid \omega \omega^{\prime}\right)\right]\left(\omega 1\left|+\int d \omega \int d \omega^{\prime}\left[V_{\omega} \mid 1 \omega^{\prime}\right)-V_{\omega^{\prime}}\right| \omega 1\right)\right]\left(\omega \omega^{\prime} \mid\right.
\end{aligned}
$$

From equation (55) and the orthogonality conditions (54), the generalized states and observables defined in equations (51) and (47) form a complete biorthogonal set of generalized eigenvectors of the "free" superoperator $\mathbb{L}^{0}$

$$
\begin{aligned}
& \left.\left.\left.\left.\left.\left.\stackrel{0}{\mathbb{L}} \mid 1)=\stackrel{0}{\mathbb{L}} \mid \omega)=0, \quad \mathbb{L}^{\mathbb{L}} \mid 1 \omega^{\prime}\right)=\left(\Omega-\omega^{\prime}\right) \mid 1 \omega^{\prime}\right), \quad \stackrel{0}{\mathbb{L}} \mid \omega 1\right)=(\omega-\Omega) \mid \omega 1\right), \quad \stackrel{0}{\mathbb{L}} \mid \omega \omega^{\prime}\right)=\left(\omega-\omega^{\prime}\right) \mid \omega \omega^{\prime}\right), \\
& \left(1 \mid \stackrel{0}{\mathbb{L}}=\left(\omega \mid \stackrel{0}{\mathbb{L}}=0, \quad\left(1 \omega^{\prime} \mid \stackrel{0}{\mathbb{L}}=\left(\Omega-\omega^{\prime}\right)\left(1 \omega^{\prime} \mid, \quad\left(\omega 1 \mid \stackrel{0}{\mathbb{L}}=(\omega-\Omega)\left(\omega 1 \mid, \quad\left(\omega \omega^{\prime} \mid \stackrel{0}{\mathbb{L}}=\left(\omega-\omega^{\prime}\right)\left(\omega \omega^{\prime} \mid\right.\right.\right.\right.\right.\right.\right.\right.
\end{aligned}
$$

If we try to construct a complete biorthogonal set of eigenvectors of the Liouville-Von Newmann superoperator $\mathbb{L}=\stackrel{0}{\mathbb{L}}+\stackrel{1}{\mathbb{L}}$, depending analytically on the small interaction parameter which we assume included in $\stackrel{1}{\mathbb{L}}$, the standard methods of perturbation theory are not applicable: the superposition of the discrete and continuous parts of the spectrum of $\mathbb{L}_{\mathbb{L}}$ will produce non defined terms in the perturbation expansion.

However, this problem can be avoided if we are satisfied, as in the pure state case, to obtain information about terms of the form $(\rho \mid O)$ or $(\rho|\mathbb{L}| O)$, considering states $\rho$ and observables $O$ for which $\left(\rho \mid \omega \omega^{\prime}\right),(\rho \mid \omega 1),\left(\rho \mid 1 \omega^{\prime}\right),\left(\omega \omega^{\prime} \mid O\right),(\omega 1 \mid O)$ and $\left(1 \omega^{\prime} \mid O\right)$ have well defined analytic extensions $\left(\rho \mid z z^{\prime}\right),(\rho \mid z 1),\left(\rho \mid 1 z^{\prime}\right),\left(z z^{\prime} \mid O\right),(z 1 \mid O)$ and $\left(1 z^{\prime} \mid O\right)$ to complex values of $z\left(z^{\prime}\right)$ on the upper (lower) complex half plane. We also consider states $\rho$ and observables $O$ for which $(\rho \mid \omega)$ and $(\omega \mid O)$ have well defined analytic extensions $(\rho \mid z)$ and $(z \mid O)$ for complex values of $z$ near $\mathbb{R}^{+}$in the upper and in the lower complex half planes. The physical bases of these analyticity properties are given in papers [6] and [7].

For these states and observables, the Cauchy theorem can be used in equation (52) to write

$$
\begin{aligned}
(\rho \mid O)= & (\rho \mid 1)(1 \mid O)+\int_{0}^{\infty} d \omega(\rho \mid \omega)(\omega \mid O)+\int_{\Gamma} d z \int_{\bar{\Gamma}} d z^{\prime}\left(\rho \mid z z^{\prime}\right)\left(z z^{\prime} \mid O\right)+ \\
& +\int_{\Gamma} d z(\rho \mid z 1)(z 1 \mid O)+\int_{\bar{\Gamma}} d z^{\prime}\left(\rho \mid 1 z^{\prime}\right)\left(1 z^{\prime} \mid O\right)
\end{aligned}
$$

where $\Gamma$ is a curve in the lower complex half plane, as shown in Fig. 1, and $\bar{\Gamma}$ is the conjugate curve in the upper complex half plane. Therefore

$$
\left.(\rho \mid O)=\left(\rho\left|\mathbb{I}_{e x t}\right| O\right), \quad \mathbb{I}_{e x t} \doteq \mid 1\right)\left(1\left|+\int d \omega\right| \omega\right)\left(\omega\left|+\int_{\bar{\Gamma}} d z \int_{\Gamma} d z^{\prime}\right| z z^{\prime}\right)\left(z z^{\prime}\left|+\int_{\bar{\Gamma}} d z\right| z 1\right)\left(z 1\left|+\int_{\Gamma} d z^{\prime}\right| 1 z^{\prime}\right)\left(1 z^{\prime} \mid .\right.
$$

If in the expression for $\mathbb{I}_{\text {ext }}$ given in equation (59), the term $\left.\int d \omega \mid \omega\right)\left(\omega \mid\right.$ is replaced by $\left.\int_{\bar{\Gamma}} d z \mid z\right)\left(z \mid\right.$ or by $\left.\int_{\Gamma} d z^{\prime} \mid z^{\prime}\right)\left(z^{\prime} \mid\right.$, the identity $(\rho \mid O)=\left(\rho\left|\mathbb{I}_{\text {ext }}\right| O\right)$ is also verified for the restricted classes of states and observables with the well defined analytic extensions defined above.

We can also define $\mathbb{L}_{\text {ext }}=\stackrel{0}{\mathbb{L}}_{\text {ext }}+\stackrel{1}{\mathbb{L}}_{\text {ext }}$, where

$$
\begin{aligned}
\stackrel{\mathbb{L}}{L} \text { ext }^{0} & \left.\int_{\Gamma} d z^{\prime}\left(\Omega-z^{\prime}\right) \mid 1 z^{\prime}\right)\left(1 z^{\prime}\left|+\int_{\bar{\Gamma}} d z(z-\Omega)\right| z 1\right)\left(z 1\left|+\int_{\bar{\Gamma}} d z \int_{\Gamma} d z^{\prime}\left(z-z^{\prime}\right)\right| z z^{\prime}\right)\left(z z^{\prime} \mid,\right. \\
\stackrel{\mathbb{L}}{\text { ext }}^{\prime} & \left.\int_{\bar{\Gamma}} d z V_{z} \mid z 1\right)\left(1\left|-\int_{\Gamma} d z^{\prime} V_{z^{\prime}}\right| 1 z^{\prime}\right)\left(1\left|+\int_{\Gamma} d z^{\prime} V_{z^{\prime}}\right| 1 z^{\prime}\right)\left(z^{\prime}\left|-\int_{\bar{\Gamma}} d z V_{z}\right| z 1\right)(z \mid+ \\
& \left.-\int_{\Gamma} d z^{\prime} V_{z^{\prime}} \mid 1\right)\left(1 z^{\prime}\left|+\int_{\bar{\Gamma}} d z \int_{\Gamma} d z^{\prime} V_{z}\right| z z^{\prime}\right)\left(1 z^{\prime}\left|+\int_{\bar{\Gamma}} d z V_{z}\right| 1\right)(z 1 \mid- \\
& \left.-\int_{\bar{\Gamma}} d z \int_{\Gamma} d z^{\prime} V_{z^{\prime}} \mid z z^{\prime}\right)\left(z 1\left|+\int_{\bar{\Gamma}} d z \int_{\Gamma} d z^{\prime} V_{z}\right| 1 z^{\prime}\right)\left(z z^{\prime}\left|-\int_{\bar{\Gamma}} d z \int_{\Gamma} d z^{\prime} V_{z^{\prime}}\right| z 1\right)\left(z z^{\prime} \mid\right.
\end{aligned}
$$


The superoperators $\stackrel{0}{\mathbb{L}}$ ext $_{\text {and }} \stackrel{1}{\mathbb{L}}$ ext $_{\text {verify }}(\rho|\stackrel{0}{\mathbb{L}}| O)=(\rho \mid \stackrel{0}{\mathbb{L}}$ ext $\mid O)$ and $(\rho|\stackrel{1}{\mathbb{L}}| O)=\left(\rho\left|\stackrel{1}{\mathbb{L}}_{\text {ext }}\right| O\right)$. The generalized

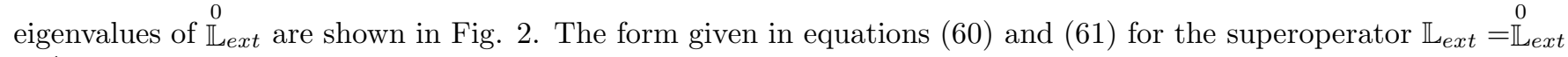
$+\stackrel{1}{\mathbb{L}}_{\text {ext }}$ can also be obtained directly from the definition $\mathbb{L}_{e x t} O \doteq H_{\bar{\Gamma}} O-O H_{\Gamma}$, where $H_{\Gamma}$ was defined in section II.

For the right eigenvalue problem $\left.\left.\mathbb{L}_{e x t} \mid \Phi\right)=\lambda \mid \Phi\right)$ we assume an expansion

$$
\mid \Phi)=\mid \stackrel{0}{\Phi})+\mid \stackrel{1}{\Phi})+\mid \stackrel{2}{\Phi})+\cdots \cdot, \quad \lambda=\stackrel{0}{\lambda}+\stackrel{1}{\lambda}+\stackrel{2}{\lambda}+\cdots \cdot \cdot
$$

with respect to the small interaction parameter that we suppose it is contained in $\stackrel{1}{\mathbb{L}}$. The following set of equations is obtained order by order

$$
\begin{aligned}
& \left.\left(\begin{array}{l}
0 \\
\lambda
\end{array}-\stackrel{0}{L}_{\text {ext }}\right) \mid \stackrel{0}{\Phi}\right)=0 \\
& \left.\left.\left(\begin{array}{l}
0 \\
\lambda
\end{array}-\mathbb{L}_{\text {ext }}\right) \mid \stackrel{1}{\Phi}\right)=\left(\begin{array}{l}
1 \\
\mathbb{L}_{\text {ext }}-\lambda
\end{array}\right) \mid \stackrel{0}{\Phi}\right)
\end{aligned}
$$

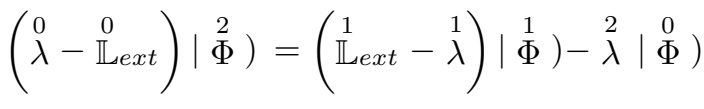

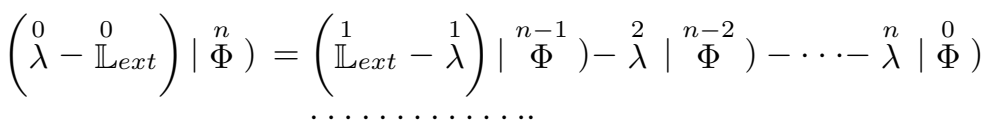

For the left eigenvalue problem $\left(\Psi \mid \mathbb{L}_{e x t}=\lambda(\Psi \mid\right.$, the expansion

$$
(\Psi)=(\stackrel{0}{\Psi} \mid+(\stackrel{1}{\Psi}|+\stackrel{2}{\Psi}|+\cdots, \quad \lambda=\stackrel{0}{\lambda}+\stackrel{1}{\lambda}+\stackrel{2}{\lambda}+\cdots \cdot,
$$

gives

$$
\begin{aligned}
& \left(\stackrel{0}{\Psi} \mid\left(\begin{array}{l}
0 \\
\lambda
\end{array}-0_{\mathbb{L}}{ }_{e x t}\right)=0\right. \\
& \left(\stackrel{1}{\Psi} \mid\left(\begin{array}{l}
0 \\
\lambda
\end{array}-\stackrel{0}{\mathbb{L}} \text { ext }\right)=\left(\stackrel{0}{\Psi} \mid\left(\begin{array}{ll}
1 \\
\mathbb{L}
\end{array}\right.\right.\right. \\
& \left(\stackrel{2}{\Psi} \mid\left(\begin{array}{l}
0 \\
\lambda
\end{array}-\stackrel{0}{\mathbb{L}_{e x t}}\right)=\left(\stackrel{1}{\Psi} \mid\left(\stackrel{1}{\mathbb{L}}_{\text {ext }}-\stackrel{1}{\lambda}\right)-(\stackrel{0}{\Psi} \mid \stackrel{2}{\lambda}\right.\right. \\
& \left(\stackrel{n}{\Psi} \mid\left(\begin{array}{l}
0 \\
\lambda
\end{array}-\mathbb{L}_{\text {ext }}\right)=\left(\stackrel{n-1}{\Psi} \mid\left(\begin{array}{ll}
\mathbb{L}_{e x t} & -1 \\
\lambda
\end{array}\right)-(\stackrel{n-2}{\Psi} \mid \stackrel{2}{\lambda}-\cdots-(\stackrel{0}{\Psi} \mid \stackrel{n}{\lambda}\right.\right.
\end{aligned}
$$

It is convenient to define the following projectors

$$
\left.\mathbb{P}_{0} \doteq \mid 1\right)\left(1\left|+\int d \omega\right| \omega\right)\left(\omega\left|, \quad \mathbb{P}_{\bar{\Gamma} \Gamma} \doteq \int_{\bar{\Gamma}} d z \int_{\Gamma} d z^{\prime}\right| z z^{\prime}\right)\left(z z^{\prime}\left|, \quad \mathbb{P}_{\bar{\Gamma}} \doteq \int_{\bar{\Gamma}} d z\right| z 1\right)\left(z 1\left|, \quad \mathbb{P}_{\Gamma} \doteq \int_{\Gamma} d z^{\prime}\right| 1 z^{\prime}\right)\left(1 z^{\prime} \mid\right.
$$

These projectors commute with $\stackrel{0}{\mathbb{L}}_{\text {ext }}$ and also satisfy

$$
\mathbb{I}_{\text {ext }}=\mathbb{P}_{0}+\mathbb{P}_{\bar{\Gamma} \Gamma}+\mathbb{P}_{\bar{\Gamma}}+\mathbb{P}_{\Gamma}, \quad \mathbb{P}_{0} \stackrel{0}{L}_{\text {ext }}=\stackrel{0}{L}_{\text {ext }} \mathbb{P}_{0}=0 .
$$

Therefore $\mathbb{P}_{0}$ is the projector on the invariant space of the time evolution generated by the superoperator $\mathbb{L}_{\text {ext }}$. As in the pure state case we will compute the discrete and the continuous spectrum.

\section{A. The discrete spectrum.}

We first consider equations (62)- $(65)$ with 


$$
\left.\left.\left.\stackrel{0}{\lambda}=0, \quad \mid \stackrel{0}{\Phi})=\mathbb{P}_{0} \mid \stackrel{0}{\Phi}\right)=\mid 1\right)(1 \mid \stackrel{0}{\Phi})+\int d \omega \mid \omega\right)(\omega \mid \stackrel{0}{\Phi}) .
$$

Multiplying equation $(63)$ by $\mathbb{P}_{0}$ and by $\mathbb{Q}_{0} \doteq \mathbb{I}_{\text {ext }}-\mathbb{P}_{0}$, we obtain

$$
\begin{aligned}
\lambda & \left.\stackrel{0}{\Phi})=\mathbb{P}_{0} \stackrel{0}{\mathbb{L}_{e x t}} \mathbb{P}_{0} \mid \stackrel{0}{\Phi}\right) \\
\left.\mathbb{Q}_{0} \mid \stackrel{1}{\Phi}\right) & \left.=\frac{(-1)}{\mathbb{Q}_{0} \stackrel{0}{\mathbb{L}_{e x t}} \mathbb{Q}_{0}} \mathbb{Q}_{0} \mathbb{L}_{\text {ext }}^{1} \mid \stackrel{0}{\Phi}\right)
\end{aligned}
$$

Equation (64) gives

$$
\begin{aligned}
& \left.\stackrel{2}{\lambda} \mid \stackrel{0}{\Phi})=\left[\mathbb{P}_{0} \stackrel{1}{\mathbb{L}} \text { ext } \frac{(-1)}{\mathbb{Q}_{0} \stackrel{0}{L}_{\text {ext }} \mathbb{Q}_{0}} \mathbb{Q}_{0} \stackrel{1}{\mathbb{L}_{\text {ext }}} \mathbb{P}_{0}\right] \mid \stackrel{0}{\Phi}\right), \\
& \left.\left.\mathbb{Q}_{0} \mid \stackrel{2}{\Phi}\right)=\frac{(-1)}{\mathbb{Q}_{0} \stackrel{0}{\mathbb{L}_{\text {ext }}} \mathbb{Q}_{0}} \mathbb{Q}_{0}\left[\frac{1}{\mathbb{L}_{e x t}}-\stackrel{1}{\lambda}\right] \mid \stackrel{1}{\Phi}\right) .
\end{aligned}
$$

From the definitions $(\sqrt{70})$ and $(61)$ we have $\mathbb{P}_{0} \stackrel{0}{\mathbb{L}}$ ext $\mathbb{P}_{0}=0$ and therefore $\stackrel{1}{\lambda}=0$ : the degeneration of the space expanded by the projector $\mathbb{P}_{0}$ is not eliminated by first order corrections. The previous equations give no information about $\left.\mathbb{P}_{0} \mid \stackrel{n}{\Phi}\right)(n=1,2, \ldots)$, and we make the usual choice $\left.\left.\mid \stackrel{n}{\Phi}\right)=\mathbb{Q}_{0} \mid \stackrel{n}{\Phi}\right)(n=1,2, \ldots)$.

But the degeneration is partially removed with the second order correction. Equation (74), an eigenvalue problem for $\stackrel{2}{\lambda}$, gives

$$
\left.\left.\left.\left.2 \pi i V_{\Omega}^{2} \mid 1\right)(1 \mid \stackrel{0}{\Phi})-2 \pi i V_{\Omega}^{2} \mid 1\right)(\omega=\Omega \stackrel{0}{\Phi})=\stackrel{2}{\lambda} \mid 1\right)(1 \mid \stackrel{0}{\Phi})+\stackrel{2}{\lambda} \int d \omega \mid \omega\right)(\omega \mid \stackrel{0}{\Phi}) .
$$

This equation has the solutions

$$
\left.\left.\left.\left.\left.\stackrel{2}{\lambda_{d}}=2 \pi i V_{\Omega}^{2}, \quad \stackrel{0}{\Phi}_{d}\right)=\mid 1\right), \quad \stackrel{2}{\lambda}_{\omega}=0, \quad \mid \stackrel{0}{\Phi} \omega\right)=\delta(\omega-\Omega) \mid 1\right)+\mid \omega\right) .
$$

We give in appendix A the details of these calculations.

For the left eigenvalue problem with

$$
\stackrel{0}{\lambda}=0, \quad \stackrel{0}{\Psi} \mid=\left(\stackrel{0}{\Psi} \mid \mathbb{P}_{0}=(\stackrel{0}{\Psi} \mid 1)\left(1 \mid+\int d \omega(\stackrel{0}{\Psi} \mid \omega)(\omega \mid,\right.\right.
$$

equations (66)-(69) give

$$
\begin{aligned}
& \left(\stackrel{0}{\Psi} \mid \stackrel{1}{\lambda}=\left(\stackrel{0}{\Psi} \mid\left(\mathbb{P}_{0} \stackrel{0}{\mathbb{L}} \text { ext } \mathbb{P}_{0}\right),\right.\right. \\
& \left(\stackrel{1}{\Psi} \mid \mathbb{Q}_{0}=\left(\stackrel{0}{\Psi} \mid \stackrel{1}{\mathbb{L}} \text { ext } \mathbb{Q}_{0} \frac{(-1)}{\mathbb{Q}_{0} \stackrel{0}{L} \text { ext }_{\mathbb{Q}_{0}}},\right.\right. \\
& \left(\stackrel{0}{\Psi} \mid \stackrel{2}{\lambda}=\left(\stackrel{0}{\Psi} \mid\left[\mathbb{P}_{0} \stackrel{1}{\mathbb{L}} \text { ext } \frac{(-1)}{\mathbb{Q}_{0} \stackrel{0}{\mathbb{L}} \text { ext } \mathbb{Q}_{0}} \mathbb{Q}_{0} \stackrel{1}{\mathbb{L}} \text { ext } \mathbb{P}_{0}\right],\right.\right. \\
& \left(\stackrel{2}{\Psi} \mid \mathbb{Q}_{0}=\left(\stackrel{1}{\Psi} \mid\left[\mathbb{1}_{\text {Lext }}-\stackrel{1}{\lambda}\right] \mathbb{Q}_{0} \frac{(-1)}{\mathbb{Q}_{0} \mathbb{L}_{\text {ext }} \mathbb{Q}_{0}},\right.\right.
\end{aligned}
$$

Once again, the degeneration is not removed by the first order corrections, and $\stackrel{1}{\lambda}=0$ as a consequence of $\mathbb{P}_{0} \stackrel{0}{\mathbb{L}}$ ext $\mathbb{P}_{0}=0$. As there is no information about $\left(\stackrel{n}{\Psi} \mid \mathbb{P}_{0}\right.$ for $n \geq 1$, the usual condition $\stackrel{n}{\Psi} \mid=\left(\stackrel{n}{\Psi} \mid \mathbb{Q}_{0}\right.$ can be imposed. For the eigenvalue problem (81) we obtain

$$
\left.2 \pi i V_{\Omega}^{2} \stackrel{0}{\Psi} \mid 1\right)\left(1 \mid-2 \pi i V_{\Omega}^{2}(\stackrel{0}{\Psi} \mid 1)\left(\omega=\Omega \mid=\stackrel{2}{\lambda}(\stackrel{0}{\Psi} \mid 1)\left(1 \mid+\stackrel{2}{\lambda} \int d \omega(\stackrel{0}{\Psi} \mid \omega)(\omega \mid .\right.\right.\right.
$$


This equation has the solutions

$$
\stackrel{2}{\lambda_{d}}=2 \pi i V_{\Omega}^{2}, \quad\left(\stackrel{0}{\Psi}_{d} \mid=\left(1\left|-(\omega=\Omega), \quad \stackrel{2}{\lambda}_{\omega}=0, \quad \stackrel{0}{\Psi_{\omega}}\right|=(\omega) .\right.\right.
$$

(See appendix A for the details).

The right and left eigenvectors given in equations (77) and (84) form a complete biorthogonal system for the $\mathbb{P}_{0}$ subspace, i.e.

$$
\begin{aligned}
& \left(\stackrel{0}{\Psi}, \mid \stackrel{0}{\Phi}_{d}\right)=1, \quad\left(\stackrel{0}{\Psi} \omega \mid \stackrel{0}{\Phi} \omega_{\omega^{\prime}}\right)=\delta\left(\omega-\omega^{\prime}\right), \quad\left(\stackrel{0}{\Psi}_{d} \mid \stackrel{0}{\Phi} \omega_{\omega^{\prime}}\right)=\left(\stackrel{0}{\Psi} \mid \stackrel{0}{\Phi}_{d}\right)=0 \\
& \left.\mathbb{P}_{0}=\mid \stackrel{0}{\Phi}\right)\left(\stackrel{0}{\Psi}_{d}\left|+\int d \omega\right| \stackrel{0}{\Phi} \omega\right)(\stackrel{0}{\Psi} \omega \mid
\end{aligned}
$$

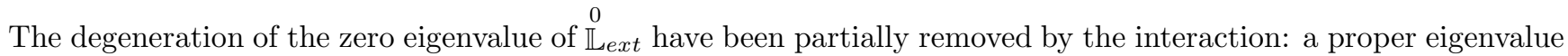
$\lambda_{d} \cong 2 \pi i V_{\Omega}^{2}$ with a single eigenvector is obtained. However, the infinite degeneration of the eigenvalue $\lambda_{\omega}=0$ $\left(\omega \in \mathbb{R}^{+}\right)$remains. This is a new feature that must be taken in consideration: the infinitely degenerated eigenvalue corresponds to the invariant states. As we see from equations (84), these invariant states are expanded, up to zero order, by the singular states $\left(\stackrel{0}{\Psi}_{\omega}\right)=(\omega)$. These invariant states only appear if we introduce the singular structure for states and observables. The singular structure is also necessary for the description of the decay of the unstable discrete state. As we will see below, the imaginary eigenvalue $\lambda_{d}$ is associated with the exponential approximation of the decay process.

\section{B. The continuous spectrum.}

Let us consider the following generalized eigenvalues and eigenvectors of $\stackrel{0}{L}_{e x t}$

$$
\left.\left.\stackrel{0}{\lambda}_{u 1}=u-\Omega, \quad \mid \stackrel{0}{\Phi}_{u 1}\right)=\mid u 1\right), \quad\left(\stackrel{0}{\Psi}_{u 1} \mid=(u 1 \mid, \quad u \in \Gamma,\right.
$$

Equation (63) gives

$$
(u-\Omega-\stackrel{0}{\mathbb{L}} \text { ext }) \mid \stackrel{1}{\Phi})=(\stackrel{1}{\mathbb{L}} \text { ext }-\stackrel{1}{\lambda}) \mid u 1) .
$$

When this equation is multiplied from the left by $(v 1 \mid$ we obtain

$$
(u-v)(v 1 \mid \stackrel{1}{\Phi})=-\stackrel{1}{\lambda} \delta_{\bar{\Gamma}}(u-v),
$$

where $\delta_{\bar{\Gamma}}(u-v)$ is the " $\delta$-function" on the curve $\bar{\Gamma}\left(\int_{\bar{\Gamma}} d u \delta_{\bar{\Gamma}}(u-v) f(u)=f(v)\right)$. From this last equation we obtain $\stackrel{1}{\lambda}_{u 1}=0$. The first order correction for the right eigenvector can be obtained from equation 86

$$
\left.\left.\left.\mid \stackrel{1}{\Phi}{ }_{u 1}\right)=\frac{V_{u}}{u-\Omega} \mid 1\right)-\int_{\Gamma} d u^{\prime} \frac{V_{u^{\prime}}}{u^{\prime}-\Omega} \mid u u^{\prime}\right)
$$

From equation (67) we obtain the first order correction of the left eigenvector with the same eigenvalue

$$
\left(\stackrel{1}{\Psi}_{u 1} \mid=\frac{V_{u}}{u-\Omega}\left[\left(1 \mid-(u \mid]-\int_{\Gamma} d u^{\prime} \frac{V_{u^{\prime}}}{u^{\prime}-\Omega}\left(u u^{\prime} \mid .\right.\right.\right.\right.
$$

The second order correction to the eigenvalue can be obtained multiplying equation (64) by ( $v 1 \mid$ from the left

$$
\begin{aligned}
(u-v)(v 1 \mid \stackrel{2}{\Phi} u 1) & =(v 1|\stackrel{1}{\mathbb{L}} \operatorname{ext}| \stackrel{1}{\Phi})-\stackrel{2}{\lambda}_{u 1} \delta_{\bar{\Gamma}}(u-v)= \\
& =\left[\int_{\Gamma} d u^{\prime} \frac{V_{u^{\prime}}^{2}}{u^{\prime}-\Omega}-\stackrel{2}{\lambda} u 1\right] \delta_{\bar{\Gamma}}(u-v)+\frac{V_{u} V_{v}}{u-\Omega} .
\end{aligned}
$$

From this equation we obtain 


$$
\stackrel{2}{\lambda} u 1_{u}=\int_{\Gamma} d u^{\prime} \frac{V_{u^{\prime}}^{2}}{u^{\prime}-\Omega}=\int_{0}^{\infty} d \omega \frac{V_{\omega}^{2}}{\omega-i 0-\Omega}=i \pi V_{\Omega}^{2}+\int_{0}^{\infty} d \omega V_{\omega}^{2} \mathcal{P}\left(\frac{1}{\omega-\Omega}\right)
$$

Therefore, the generalized eigenvalues of $\stackrel{0}{L}$ ext $^{0}$, represented by points $z=u-\Omega(u \in \bar{\Gamma})$ of the complex plane, are shifted to the upper half plane by the interaction. The corresponding eigenvalues of $\mathbb{L}_{e x t}$ are the points $z=u-\Omega+\stackrel{2}{\lambda}_{u 1}$, where $u \in \bar{\Gamma}$ and $\stackrel{2}{\lambda}_{u 1}$ is given by equation (89) (see Fig. 2).

We can also consider the following generalized eigenvalues and eigenvectors of $\stackrel{0}{L}$ ext $^{0}$

$$
\left.\left.\stackrel{0}{\lambda}_{1 u^{\prime}}=\Omega-u^{\prime}, \quad \mid \stackrel{0}{\Phi}_{1 u^{\prime}}\right)=\mid 1 u^{\prime}\right), \quad\left(\stackrel{0}{\Psi}_{1 u^{\prime}} \mid=\left(1 u^{\prime} \mid, \quad u^{\prime} \in \Gamma,\right.\right.
$$

The perturbation method gives

$$
\begin{aligned}
\stackrel{1}{\lambda}_{1 u^{\prime}} & =0 \\
\stackrel{2}{\lambda}_{1 u^{\prime}} & =\int_{\bar{\Gamma}} d u \frac{V_{u}^{2}}{\Omega-u}=i \pi V_{\Omega}^{2}-\int_{0}^{\infty} d \omega V_{\omega}^{2} \mathcal{P}\left(\frac{1}{\omega-\Omega}\right), \\
\left.\mid \stackrel{1}{\Phi}_{1 u^{\prime}}\right) & \left.\left.=\frac{V_{u^{\prime}}}{u^{\prime}-\Omega} \mid 1\right)-\int_{\bar{\Gamma}} d u \frac{V_{u}}{u-\Omega} \mid u u^{\prime}\right), \\
\left(\stackrel{1}{\Psi}_{1 u^{\prime}} \mid\right. & =\frac{V_{u^{\prime}}}{u^{\prime}-\Omega}\left[\left(1 \mid-\left(u^{\prime} \mid\right]-\int_{\bar{\Gamma}} d u \frac{V_{u}}{u-\Omega}\left(u u^{\prime} \mid .\right.\right.\right.
\end{aligned}
$$

This result shows that the generalized eigenvalues of equation (90) are also shifted to the upper half plane by the interaction (see Fig. 2).

Finally, we can consider the following generalized eigenvalues and eigenvectors of $\mathbb{L}_{\text {ext }}$

$$
\left.\left.{\stackrel{0}{\lambda} u u^{\prime}}=u-u^{\prime}, \quad \mid \stackrel{0}{\Phi}_{u u^{\prime}}\right)=\mid u u^{\prime}\right), \quad\left(\stackrel{0}{\Psi}_{u u^{\prime}} \mid=\left(u u^{\prime} \mid, \quad u \in \bar{\Gamma}, u^{\prime} \in \Gamma\right.\right.
$$

(see the shaded region in Fig.1). In this case the perturbed expansion gives

$$
\begin{aligned}
\stackrel{1}{\lambda}_{u u^{\prime}} & =\stackrel{2}{\lambda}_{u u^{\prime}}=0, \\
\left.\mid \stackrel{1}{\Phi}_{u u^{\prime}}\right) & \left.\left.=\frac{V_{u}}{u-\Omega} \mid 1 u^{\prime}\right)+\frac{V_{u^{\prime}}}{u^{\prime}-\Omega} \mid u 1\right), \\
\left(\stackrel{1}{\Psi}_{u u^{\prime}} \mid\right. & =\frac{V_{u}}{u-\Omega}\left(1 u^{\prime} \mid+\frac{V_{u^{\prime}}}{u^{\prime}-\Omega}(u 1 \mid .\right.
\end{aligned}
$$

There is no shift of this part of the generalized spectrum by the interaction.

\section{Time evolution.}

In the previous subsection we obtained by a perturbation method a set of generalized eigenvalues and eigenvectors of the superoperator $\mathbb{L}_{e x t}$, which reduce to the complete biorthogonal set of $\mathbb{L}_{e x t}$ when the parameter of the interaction goes to zero. The generalized eigenvalues of $\mathbb{L}_{e x t}$ are shown in Fig. 3. It seems reasonable to assume that the obtained generalized eigenvalues and eigenvectors of $\mathbb{L}_{e x t}$, also form a complete biorthogonal set, at least for small values of the interaction parameter 2 . If this is the case, the time evolution operator is

$$
\begin{aligned}
\exp \left(i \mathbb{L}_{e x t} t\right)= & \left.\int d \omega \exp \left(i \lambda_{\omega} t\right) \mid \Phi_{\omega}\right)\left(\Psi_{\omega}\left|+\exp \left(i \lambda_{d} t\right)\right| \Phi_{d}\right)\left(\Psi_{d}\left|+\int_{\bar{\Gamma}} d u \exp \left(i \lambda_{u 1} t\right)\right| \Phi_{u 1}\right)\left(\Psi_{u 1} \mid+\right. \\
& \left.+\int_{\Gamma} d u^{\prime} \exp \left(i \lambda_{1 u^{\prime}} t\right) \mid \Phi_{1 u^{\prime}}\right)\left(\Psi_{1 u^{\prime}}\left|+\int_{\bar{\Gamma}} d u \int_{\Gamma} d u^{\prime} \exp \left(i \lambda_{u u^{\prime}} t\right)\right| \Phi_{u u^{\prime}}\right)\left(\Psi_{u u^{\prime}} \mid .\right.
\end{aligned}
$$

\footnotetext{
${ }^{2}$ This fact can be explicitly verified up to second order for the generalized eigenvectors obtained in sections IIIA and IIIB.
} 
Up to second order, the eigenvalues are

$$
\begin{aligned}
\lambda_{\omega} & =0, \\
\lambda_{d} & =2 \pi i V_{\Omega}^{2}, \\
\lambda_{u 1} & =u-\Omega+i \pi V_{\Omega}^{2}+\int_{0}^{\infty} d \omega V_{\omega}^{2} \mathcal{P}\left(\frac{1}{\omega-\Omega}\right), \\
\lambda_{1 u^{\prime}} & =\Omega-u^{\prime}+i \pi V_{\Omega}^{2}-\int_{0}^{\infty} d \omega V_{\omega}^{2} \mathcal{P}\left(\frac{1}{\omega-\Omega}\right), \\
\lambda_{u u^{\prime}} & =u-u^{\prime}
\end{aligned}
$$

and the approximated expressions for the eigenvectors are given in the previous subsection.

The time evolution of the mean value of an observable $O$, given by

$$
\langle O\rangle_{\rho_{t}}=\left(\rho_{t} \mid O\right)=\left(\rho_{0}|\exp (i \mathbb{L} t)| O\right),
$$

can be easily computed using (94). Let us consider the time evolution of the unstable state given by $\left(\rho_{0} \mid=(1)\right.$. If we call by $\varepsilon$ the small multiplicative interaction parameter included in $V_{\omega}$, we can use the explicit approximated expressions of the eigenvectors to prove that

$$
\begin{aligned}
\left(1 \mid \Phi_{\omega}\right)\left(\Psi_{\omega} \mid 1\right) & =O\left(\varepsilon^{2}\right), \\
\left(1 \mid \Phi_{d}\right)\left(\Psi_{d} \mid 1\right) & =1+O\left(\varepsilon^{2}\right), \\
\left(1 \mid \Phi_{u 1}\right)\left(\Psi_{u 1} \mid 1\right) & =O\left(\varepsilon^{2}\right), \\
\left(1 \mid \Phi_{1 u^{\prime}}\right)\left(\Psi_{1 u^{\prime}} \mid 1\right) & =O\left(\varepsilon^{2}\right), \\
\left(1 \mid \Phi_{u u^{\prime}}\right)\left(\Psi_{u u^{\prime}} \mid 1\right) & =O\left(\varepsilon^{2}\right),
\end{aligned}
$$

and therefore the survival probability of the unstable state which is represented by the state functional $\left(\rho_{0}\right)=(1)$ (and also by the vector state $|1\rangle$ ) is approximately given by

$$
\left(\rho_{t} \mid 1\right)=(1|\exp (i \mathbb{L} t)| 1) \cong \exp \left(-2 \pi V_{\Omega}^{2} t\right) .
$$

This expression for the survival probability is valid if $\varepsilon^{2} \ll 1$ and $t \leq \varepsilon^{-2}$.

Taking into account that

$$
\begin{aligned}
\left(1 \mid \Phi_{\omega^{\prime}}\right)\left(\Psi_{\omega^{\prime}} \mid \omega\right) & =\delta\left(\omega^{\prime}-\Omega\right) \delta\left(\omega^{\prime}-\omega\right)+O\left(\varepsilon^{2}\right), \\
\left(1 \mid \Phi_{d}\right)\left(\Psi_{d} \mid \omega\right) & =-\delta(\omega-\Omega)+O\left(\varepsilon^{2}\right), \\
\left(1 \mid \Phi_{u 1}\right)\left(\Psi_{u 1} \mid \omega\right) & =O\left(\varepsilon^{2}\right), \\
\left(1 \mid \Phi_{1 u^{\prime}}\right)\left(\Psi_{1 u^{\prime}} \mid \omega\right) & =O\left(\varepsilon^{2}\right), \\
\left(1 \mid \Phi_{u u^{\prime}}\right)\left(\Psi_{u u^{\prime}} \mid \omega\right) & =O\left(\varepsilon^{2}\right),
\end{aligned}
$$

for the initial unstable state $\left(\rho_{0} \mid=(1 \mid\right.$ we obtain

$$
\left(\rho_{t} \mid \omega\right)=(1|\exp (i \mathbb{L} t)| \omega) \cong \delta(\omega-\Omega)\left[1-\exp \left(-2 \pi V_{\Omega}^{2} t\right)\right],
$$

also valid if $\varepsilon^{2} \ll 1$ and $t \leq \varepsilon^{-2}$. Equations (96)-(97) describes the transition of the unstable state (1|, into $(\omega=\Omega \mid$.

Therefore, the obtained set of generalized eigenvectors is a useful tool for a complete description of the decay process. The pure state $|1\rangle$ is given in this formalism by the state functional (1|, and the standard approximated expression its the survival probability is reobtained in equation (96). But in addition we obtain in equation (97) an explicit expression for the by-products of the decay process.

\section{CONCLUSIONS.}

When faced with the problem of the spectral decomposition of the Hamiltonian

$$
\begin{aligned}
& H=\stackrel{0}{H}+\stackrel{1}{H}, \\
& \stackrel{0}{H}=\Omega|1\rangle\left\langle 1\left|+\int_{0}^{\infty} d \omega \omega\right| \omega\right\rangle\langle\omega|, \quad \Omega \in \mathbb{R}^{+} \\
& \stackrel{1}{H}=\int_{0}^{\infty} d \omega V_{\omega}|\omega\rangle\left\langle 1\left|+\int_{0}^{\infty} d \omega \overline{V_{\omega}}\right| 1\right\rangle\left\langle\omega\left|+\int_{0}^{\infty} d \omega \int_{0}^{\infty} d \omega^{\prime} V_{\omega \omega^{\prime}}\right| \omega\right\rangle\left\langle\omega^{\prime}\right|,
\end{aligned}
$$


we find that the superposition of the continuous and the discrete part of the spectrum of $\stackrel{0}{H}$ makes impossible to apply the standard methods of perturbation theory.

If we restrict the class of states (observables) of the system to the set represented by vectors $\Phi(\Psi)$ for which $\langle\omega \mid \Phi\rangle$ $(\langle\omega \mid \Psi\rangle)$ has a well defined analytic extension $\langle z \mid \Phi\rangle(\langle z \mid \Psi\rangle)$ to the lower (upper) complex half plane, it is possible to decompose the amplitude $\langle\Psi \mid \Phi\rangle$ of the state $|\Phi\rangle$ to be observed in the state $|\Psi\rangle$ in the form

$$
\langle\Psi \mid \Phi\rangle=\left\langle\Psi\left|I_{\Gamma}\right| \Phi\right\rangle, \quad I_{\Gamma} \doteq|1\rangle\left\langle 1\left|+\int_{\Gamma} d z\right| z\right\rangle\langle z|,
$$

being $\Gamma$ a curve in the lower complex half plane as shown in fig.1.

For the amplitude of the time evolved state $\left|\Phi_{t}\right\rangle=\exp (-i H t)|\Phi\rangle$ to be observed in $|\Psi\rangle$, we also have

$$
\left\langle\Psi \mid \Phi_{t}\right\rangle=\langle\Psi|\exp (-i H t)| \Phi\rangle=\left\langle\Psi\left|\exp \left(-i H_{\Gamma} t\right)\right| \Phi\right\rangle
$$

where

$$
\begin{aligned}
H_{\Gamma} & =\stackrel{0}{H}_{\Gamma}+\stackrel{1}{H}_{\Gamma} \\
\stackrel{0}{H}_{\Gamma} & \doteq \Omega|1\rangle\left\langle 1\left|+\int_{\Gamma} d z z\right| z\right\rangle\langle z| \\
\stackrel{1}{H} & \doteq \int_{\Gamma} d z V_{z}|z\rangle\left\langle 1\left|+\int_{\Gamma} d z \overline{V_{\bar{z}}}\right| 1\right\rangle\left\langle z\left|+\int_{\Gamma} d z \int_{\Gamma} d z^{\prime} V_{z z^{\prime}}\right| z\right\rangle\left\langle z^{\prime}\right|
\end{aligned}
$$

Starting from the right (left) eigenvectors $|1\rangle$ and $|z\rangle(\langle 1|$ and $\langle z|)$, which form a complete biorthogonal set for the spectral decomposition of $\stackrel{0}{H}{ }_{\Gamma}$, it is possible to obtain a well defined perturbative algorithm to compute the corresponding right (left) eigenvectors $\left|f_{\Omega}\right\rangle$ and $\left|f_{z}\right\rangle\left(\left\langle\tilde{f}_{\Omega}\right|\right.$ and $\left.\left\langle\tilde{f}_{z}\right|\right)$, a complete biorthogonal set for the spectral decomposition of $H_{\Gamma}$, i.e.

$$
\begin{gathered}
I_{\Gamma}=\left|f_{\Omega}\right\rangle\left\langle\tilde{f}_{\Omega}\left|+\int_{\Gamma} d u\right| f_{u}\right\rangle\left\langle\tilde{f}_{u}\right| \\
H_{\Gamma}=\lambda_{\Omega}\left|f_{\Omega}\right\rangle\left\langle\widetilde{f}_{\Omega}\left|+\int_{\Gamma} d u \lambda_{u}\right| f_{u}\right\rangle\left\langle\widetilde{f}_{u}\right|, \\
\left\langle\widetilde{f}_{\Omega} \mid f_{\Omega}\right\rangle=1, \quad\left\langle\tilde{f}_{u} \mid f_{u^{\prime}}\right\rangle=\delta_{\Gamma}\left(u-u^{\prime}\right), \quad\left\langle\widetilde{f}_{\Omega} \mid f_{u}\right\rangle=\left\langle\tilde{f}_{u} \mid f_{\Omega}\right\rangle=0 .
\end{gathered}
$$

For the Hamiltonian $H_{\Gamma}$ of the general form given in equations (101), the orthogonality and completeness relations (equations (104) and (102)) can be verified by explicit calculations up to any finite order in the perturbation. For the Friedrichs model (a special case of the Hamiltonian given in equation (98)), the solutions of the eigenvalue problem obtained by the perturbation alghorithm coincide with the exact solutions obtained by Sudarshan C.B.Chiu, V.Gorini [1]. The same complex spectral decomposition for the Friedrichs model was also obtained by T.Petrosky, I.Prigogine and S.Tasaki [8], with a perturbative method using a "time ordering rule" by which an small imaginary part is included to avoid small denominators, with a different sign according to the type of transition involved. In the perturbative algorithm presented in this paper, there is no need of additional rules to avoid the singularities due to resonances between discrete and continuous parts of the unperturbed spectrum. When states and observables are restricted to have suitable analytic properties, the deformation of the integrals over the continuous part of the spectrum to a curve in the complex lower half plane, eliminate the continuous-discrete resonances. After the expressions for the eigenvalues and eigenvectors are obtained up to de desired order, the complex contour of integration can be deformed back to the real axis. In this way, the "time ordering rule" of reference [8] can be deduced from the analytic extension properties of states and observables.

If the transition probability

$$
P_{t}=|\langle\Psi|\exp (-i H t)| \Phi\rangle|^{2}=\left|\exp \left(-i \lambda_{\Omega} t\right)\left\langle\Psi \mid f_{\Omega}\right\rangle\left\langle\tilde{f}_{\Omega} \mid \Phi\right\rangle+\int_{\Gamma} d z \exp (-i z t)\left\langle\Psi \mid f_{z}\right\rangle\left\langle\tilde{f}_{z} \mid \Phi\right\rangle\right|^{2},
$$

is obtained through eigenvalues and eigenvectors computed up to n-th order, the necessary conditions for a good approximation of $P_{t}$ are $\varepsilon^{n+1} \ll 1$ and $\varepsilon^{n+1} t \ll 1$ where $\varepsilon$ is the small interaction parameter. Then, even for small interactions, it is only for not too large times that we can obtain a good approximation of $P_{t}$. 
An important advantage of the spectral decomposition in terms of generalized eigenvectors with complex eigenvalues is that it can be obtained with a well defined perturbation algorithm. The fact that this spectral decomposition is only feasible for states $|\Phi\rangle$ and observables $|\Psi\rangle$ with the analytic properties mentioned above is not a limitation. When we have to model a state or an observable from the finite amount of information coming from experimental data it is always possible to choose $\langle\omega \mid \Phi\rangle(\langle\omega \mid \Psi\rangle)$ with well defined analytic extensions to the lower (upper) complex half plane. Moreover, we are usually free to choose upper analytic extensions for the states and lower analytic extensions for the observables [5]. For this choice, we have

$$
P_{t}=|\langle\Psi|\exp (-i H t)| \Phi\rangle|^{2}=\left|\exp \left(-i \bar{\lambda}_{\Omega} t\right)\left\langle\Phi \mid \widetilde{f}_{\Omega}\right\rangle\left\langle f_{\Omega} \mid \Psi\right\rangle+\int_{\bar{\Gamma}} d z \exp (-i z t)\left\langle\Phi \mid \widetilde{f}_{z}\right\rangle\left\langle f_{z} \mid \Psi\right\rangle\right|^{2},
$$

where $\left|\widetilde{f}_{\Omega}\right\rangle$ and $\left|\widetilde{f}_{z}\right\rangle\left(\left\langle f_{\Omega}\right|\right.$ and $\left.\left\langle f_{z}\right|\right)$ are right (left) generalized eigenvectors of $H_{\bar{\Gamma}}$, being $\bar{\Gamma}$ a curve in the upper half of the complex plane.

For states and observables having both analytic extensions to the upper and lower half plane near the real axis, both expressions (105) and (106) can be used to obtain $P_{t}$. However, equation (106) would be a bad choice for $t>0$, as the positive imaginary part of the eigenvalues will give a well defined $P_{t}$ in terms of unbounded factors (the time evolution would include exponentially growing terms).

We also considered in this paper the class of observables represented by operators of the form

$$
\left.\left.\left.\left.\left.\mid O)=O_{1} \mid 1\right)+\int_{0}^{\infty} d \omega O_{\omega} \mid \omega\right)+\int_{0}^{\infty} d \omega \int_{0}^{\infty} d \omega^{\prime} O_{\omega \omega^{\prime}} \mid \omega \omega^{\prime}\right)+\int_{0}^{\infty} d \omega O_{\omega 1} \mid \omega 1\right)+\int_{0}^{\infty} d \omega^{\prime} O_{1 \omega^{\prime}} \mid 1 \omega^{\prime}\right),
$$

where the second term of the right hand side is a singular operator, and

$$
|1\rangle \doteq|1\rangle\langle 1|, \quad| \omega) \doteq|\omega\rangle\left\langle\omega|, \quad| \omega \omega^{\prime}\right) \doteq|\omega\rangle\left\langle\omega^{\prime}|, \quad| \omega 1\right) \doteq|\omega\rangle\left\langle 1|, \quad| 1 \omega^{\prime}\right) \doteq|1\rangle\left\langle\omega^{\prime}\right| .
$$

Following the work of references [9], [6] and [7], we considered the states as functionals $\rho$ acting on observables $O$ to give the mean value of the observable in the state $\left(\langle O\rangle_{\rho} \doteq(\rho \mid O)\right)$. The states can be expressed as linear combinations of the functionals defined by

$$
(1 \mid O)=O_{1}, \quad(\omega \mid O)=O_{\omega}, \quad\left(\omega \omega^{\prime} \mid O\right)=O_{\omega \omega^{\prime}}, \quad(\omega 1 \mid O)=O_{\omega 1}, \quad\left(1 \omega^{\prime} \mid O\right)=O_{1 \omega^{\prime}} .
$$

The Liouville-Von Newmann superoperators $\stackrel{0}{\mathbb{L}}=[\stackrel{0}{H}$,$] and \stackrel{1}{\mathbb{L}}=[\stackrel{1}{H}$,$] can be expanded in terms of the generalized$ observables and states given in equations (108) and (109). For the Friedrichs model we obtain

$$
\begin{aligned}
\stackrel{0}{\mathbb{L}}= & \left.\int d \omega^{\prime}\left(\Omega-\omega^{\prime}\right) \mid 1 \omega^{\prime}\right)\left(1 \omega^{\prime}\left|+\int d \omega(\omega-\Omega)\right| \omega 1\right)\left(\omega 1\left|+\int d \omega \int d \omega^{\prime}\left(\omega-\omega^{\prime}\right)\right| \omega \omega^{\prime}\right)\left(\omega \omega^{\prime} \mid,\right. \\
\stackrel{1}{\mathbb{L}}= & \left.\left.\left.\left.\int d \omega V_{\omega}[\mid \omega 1)-\mid 1 \omega\right)\right]\left(1\left|+\int d \omega V_{\omega}[\mid 1 \omega)-\right| \omega 1\right)\right]\left(\omega\left|+\int d \omega\left[-V_{\omega} \mid 1\right)+\int d \omega^{\prime} V_{\omega^{\prime}}\right| \omega^{\prime} \omega\right)\right](1 \omega \mid+ \\
& \left.\left.\left.+\int d \omega\left[V_{\omega} \mid 1\right)-\int d \omega^{\prime} V_{\omega^{\prime}} \mid \omega \omega^{\prime}\right)\right]\left(\omega 1\left|+\int d \omega \int d \omega^{\prime}\left[V_{\omega} \mid 1 \omega^{\prime}\right)-V_{\omega^{\prime}}\right| \omega 1\right)\right]\left(\omega \omega^{\prime} \mid .\right.
\end{aligned}
$$

Assuming suitable analytic properties for $\rho$ and $O$, the eigenvalue problem for $\mathbb{L}=\stackrel{0}{\mathbb{L}}+\stackrel{1}{\mathbb{L}}$ can be transformed into the eigenvalue problem for $\mathbb{L}_{e x t}$, obtained from $\mathbb{L}$ replacing the integrals $\int_{0}^{\infty} d \omega$ and $\int_{0}^{\infty} d \omega^{\prime}$ by $\int_{\bar{\Gamma}} d z$ and $\int_{\Gamma} d z^{\prime}$, being $\bar{\Gamma}(\Gamma)$ a curve in the upper (lower) complex half plane. Starting from the right and left generalized eigenvectors of $\stackrel{L}{L}_{e x t}$, a well defined perturbation algorithm can be used to obtain a complete biorthogonal set of generalized eigenvectors of $\mathbb{L}_{\text {ext }}$

$$
\begin{gathered}
\left.\mathbb{L}_{e x t}=\int d \omega \lambda_{\omega} \mid \Phi_{\omega}\right)\left(\Psi_{\omega}\left|+\lambda_{d}\right| \Phi_{d}\right)\left(\Psi_{d}\left|+\int_{\bar{\Gamma}} d u \lambda_{u 1}\right| \Phi_{u 1}\right)\left(\Psi_{u 1}\left|+\int_{\Gamma} d u^{\prime} \lambda_{1 u^{\prime}}\right| \Phi_{1 u^{\prime}}\right)\left(\Psi_{1 u^{\prime}}\left|+\int_{\bar{\Gamma}} d u \int_{\Gamma} d u^{\prime} \lambda_{u u^{\prime}}\right| \Phi_{u u^{\prime}}\right)\left(\Psi_{u u^{\prime}} \mid .\right. \\
\left.\mathbb{I}_{e x t}=\int d \omega \mid \Phi_{\omega}\right)\left(\Psi_{\omega}|+| \Phi_{d}\right)\left(\Psi_{d}\left|+\int_{\bar{\Gamma}} d u\right| \Phi_{u 1}\right)\left(\Psi_{u 1}\left|+\int_{\Gamma} d u^{\prime}\right| \Phi_{1 u^{\prime}}\right)\left(\Psi_{1 u^{\prime}}\left|+\int_{\bar{\Gamma}} d u \int_{\Gamma} d u^{\prime}\right| \Phi_{u u^{\prime}}\right)\left(\Psi_{u u^{\prime}} \mid .\right.
\end{gathered}
$$

The generalized eigenvalues of $\mathbb{L}_{e x t}$ and $\mathbb{L}_{e x t}$ are shown in Figs. 2 and 3 . The time evolution of the mean value for any observable $O$ of the form given in equation (107) can be obtained in terms of these generalized eigenvalues and 
eigenvectors. For example, it is possible to obtain the time evolution of the unstable state $\rho_{0}=\left(1 \mid\right.$. If $\varepsilon^{2} \ll 1$ and $t \leq \varepsilon^{-2}$ (being $\varepsilon$ the interaction parameter), we obtain

$$
\left(\rho_{t} \mid=\left(1 \mid \exp (i \mathbb{L} t) \cong \exp \left(-2 \pi V_{\Omega}^{2} t\right)\left(1 \mid+\left[1-\exp \left(-2 \pi V_{\Omega}^{2} t\right)\right](\omega=\Omega \mid\right.\right.\right.
$$

Therefore, the obtained set of generalized eigenvectors is a useful tool for a complete description of the decay process. The pure state $|1\rangle$ is given in this formalism by the state functional (1|, and the standard approximated expression for its survival probability is reobtained. But in addition we obtain an explicit expression for the by-products of the decay process.

The generalized spectral decomposition is useful to describe the time evolution of the physical state functionals, because the contribution of the resonances is incorporated through the exponential dumping factors. However, it is important to understand that not all the generalized left eigenvectors of the Liouville-Von Newmann superoperator $\mathbb{L}$ involved in the spectral decomposition can have an independent physical meaning: Let us consider $\left(\rho \mid=\left(\Psi_{\lambda} \mid\right.\right.$, where $\left(\Psi_{\lambda} \mid\right.$ is a left generalized eigenvector of $\mathbb{L}$ with eigenvalue $\lambda \neq 0$. Therefore we have $\left(\Psi_{\lambda} \mid \mathbb{L}=\lambda\left(\Psi_{\lambda} \mid\right.\right.$. Acting with both sides of this equation on the identity operator $I$ we obtain

$$
\lambda\left(\Psi_{\lambda} \mid I\right)=\left(\Psi_{\lambda} \mid \mathbb{L} I\right)=\left(\Psi_{\lambda} \mid H I-I H\right)=\left(\Psi_{\lambda} \mid H-H\right)=0 .
$$

If $\lambda \neq 0,\left(\Psi_{\lambda} \mid I\right)=0$ and the total probability condition $(\rho \mid I)=1$ is not verified by $\left(\Psi_{\lambda} \mid\right.$. Therefore, it can not be a physical state.

\section{ACKNOWLEDGMENTS}

This work was partially supported by Grant No. CI1-CT94-0004 of the European Community, Grant No. PID-0150 of CONICET (National Research Council of Argentina), Grant No. EX-198 of Buenos Aires University, Grant No. 12217/1 of Fundación Antorchas, and also a Grant from the Foundation pour la Recherche Foundamentale OLAM.

\section{APPENDIX A: SECOND ORDER EIGENVALUES FOR THE DISCRETE SPECTRUM.}

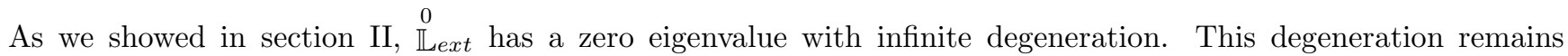
unchanged up to first order but it is partially removed in the second order approximation.

For the right eigenvalue problem, the second order corrections $\stackrel{2}{\lambda}$ to the eigenvalues is given by equation (76)

$$
\left.\left.\left.\left.2 \pi i V_{\Omega}^{2} \mid 1\right)(1 \mid \stackrel{0}{\Phi})-2 \pi i V_{\Omega}^{2} \mid 1\right)(\omega=\Omega \mid \stackrel{0}{\Phi})=\stackrel{2}{\lambda} \mid 1\right)(1 \mid \stackrel{0}{\Phi})+\stackrel{2}{\lambda} \int d \omega \mid \omega\right)(\omega \mid \stackrel{0}{\Phi}),
$$

which is equivalent to the set of equations

$$
\begin{aligned}
& \stackrel{2}{\lambda}(1 \mid \stackrel{0}{\Phi})=2 \pi i V_{\Omega}^{2}(1 \mid \stackrel{0}{\Phi})-2 \pi i V_{\Omega}^{2}(\omega=\Omega \mid \stackrel{0}{\Phi}) \\
& \stackrel{2}{\lambda}(\omega \mid \stackrel{0}{\Phi})=0, \quad \omega \in \mathbb{R}^{+} .
\end{aligned}
$$

If $\stackrel{2}{\lambda} \neq 0$, equations (A3) (A2) give $(\omega \mid \stackrel{0}{\Phi})=0$ and $\stackrel{2}{\lambda}(1 \mid \stackrel{0}{\Phi})=2 \pi i V_{\Omega}^{2}(1 \mid \stackrel{0}{\Phi})$. Therefore

$$
\left.\left.\stackrel{2}{\lambda}_{d}=2 \pi i V_{\Omega}^{2}, \quad \mid \stackrel{0}{\Phi}_{d}\right)=\mid 1\right)
$$

If $\stackrel{2}{\lambda}=0$, equation (A3) give no condition on $(\omega \mid \stackrel{0}{\Phi})$, so we can choose $(\omega \mid \stackrel{0}{\Phi})=\delta(\omega-\widetilde{\omega})$. Replacing in (A2 we obtain $(1 \mid \stackrel{0}{\Phi})=\delta(\Omega-\widetilde{\omega})$, and therefore

$$
\stackrel{2}{\lambda} \widetilde{\omega}=0, \quad \mid \stackrel{0}{\Phi} \widetilde{\omega})=\mid \widetilde{\omega})+\delta(\widetilde{\omega}-\Omega) \mid 1) .
$$

For the left eigenvectors, the second order correction to the eigenvalues is given by equation (83)

$$
2 \pi i V_{\Omega}^{2}(\stackrel{0}{\Psi} \mid 1)\left(1 \mid-2 \pi i V_{\Omega}^{2}(\stackrel{0}{\Psi} \mid 1)\left(\omega=\Omega \mid=\stackrel{2}{\lambda}(\stackrel{0}{\Psi} \mid 1)\left(1 \mid+\stackrel{2}{\lambda} \int d \omega(\stackrel{0}{\Psi} \mid \omega)(\omega \mid,\right.\right.\right.
$$

or equivalently 


$$
\begin{aligned}
& \stackrel{2}{\lambda}(\stackrel{0}{\Psi} \mid 1)=2 \pi i V_{\Omega}^{2}(\stackrel{0}{\Psi} \mid 1), \\
& \stackrel{0}{\lambda}(\stackrel{0}{\Psi} \mid \omega)=-2 \pi i V_{\Omega}^{2}(\stackrel{0}{\Psi} \mid 1) \delta(\omega-\Omega) .
\end{aligned}
$$

If $\stackrel{2}{\lambda} \neq 0$, equation (A7) gives $\stackrel{2}{\lambda} d=2 \pi i V_{\Omega}^{2}$ and no condition on $(\stackrel{0}{\Psi} \mid 1)$. If we choose $(\stackrel{0}{\Psi} \mid 1)=1$, equation $($ A 8 implies $\left(\stackrel{0}{\Psi}_{d} \mid \omega\right)=-\delta(\omega-\Omega)$. Therefore

$$
\stackrel{2}{\lambda}_{d}=2 \pi i V_{\Omega}^{2}, \quad\left(\stackrel{0}{\Psi}_{d} \mid=\left(1 \mid-\int d \omega \delta(\omega-\Omega)(\omega)=(1 \mid-(\omega=\Omega \mid\right.\right.
$$

If $\stackrel{2}{\lambda}=0$, equation (A7) implies $(\stackrel{0}{\Psi} \mid 1)=0$, while equation A8 gives no condition on $(\stackrel{0}{\Psi} \mid \omega)$. We can choose

$$
\stackrel{2}{\lambda}=0, \quad(\stackrel{0}{\Psi} \omega \mid=(\omega) .
$$

We have obtained the eigenvalues and eigenvectors quoted in equations (77) and (84). The normalization constants have been chosen to satisfy the orthogonality conditions

$$
\left(\stackrel{0}{\Psi}_{d} \mid \stackrel{0}{\Phi}_{d}\right)=1, \quad\left(\stackrel{0}{\Psi}_{\omega} \mid \stackrel{0}{\Phi}_{\omega^{\prime}}\right)=\delta\left(\omega-\omega^{\prime}\right), \quad\left(\stackrel{0}{\Psi}_{d} \mid \stackrel{0}{\Phi}_{\omega}\right)=\left(\stackrel{0}{\Psi}_{\omega} \mid \stackrel{0}{\Phi}_{d}\right)=0
$$

as can be easily verified using equations (A4), ( $\mathrm{A} 5),(\mathrm{A9})$ and $(\mathrm{A} 10)$. It is also straighforward to verify that these eigenvectors form a complete set to expand the subspace generated by $\mathbb{P}_{0}$, i.e.

$$
\left.\mathbb{P}_{0}=\mid 1\right)\left(1\left|+\int d \omega\right| \omega\right)\left(\omega|=| \stackrel{0}{\Phi_{d}}\right)\left(\stackrel{0}{\Psi}_{d}\left|+\int d \omega\right| \stackrel{0}{\Phi} \omega\right)(\stackrel{0}{\Psi} \omega \mid
$$

[1] E.C.G.Sudarshan, C.B.Chiu, V.Gorini, Phys.Rev.D, 18, 2914-2929, (1978)

[2] A.Bohm, Quantum Mechanics: Fundations and Applications, Springer-Verlag, Berlin, (1986)

[3] A.Bohm, M.Gadella, Dirac Kets, Gamov Vectors and Gel'fand Triplets, Springer-Verlag, Berlin, (1989)

[4] E.C.G.Sudarshan, Phys.Rev.A, 50, 2006-2026, (1994)

[5] M.Castagnino, R.Laura, Phys.Rev.A, 56, 108-119, (1997)

[6] R.Laura, M.Castagnino, Phys.Rev.A, 57, 4140-4152, (1997)

[7] R.Laura, M.Castagnino, Phys.Rev.E, 57, 3948-3961, (1998)

[8] T.Petrosky, I.Prigogine, S.Tasaki, Physica A, 173, 175-242, (1991)

[9] I.Antoniou, Z.Suchanecki, R.Laura, S.Tasaki, Physica A, 241, 737-772, (1997)

[10] M.Reed, B.Simon, Methods of Modern Mathematical Physics, Vol IV, Academic Press, New York, (1978)

[11] M.Hübner, H.Spohn, Rev.Math.Phys., 7, 363-387, (1995)

[12] A.Messiah, Mecánica Cuántica, Tecnos, Madrid, (1965)

[13] S.Flügge, Practical Quantum Mechanics, Spinger-Verlag, New York Heidelberg Berlin, (1974) 